\title{
1 Novel engineered high performance sugar beetroot 2D nanoplatelet-cementitious 2 composites
}

Hasan Hasan ${ }^{1}$, Bo Huang ${ }^{1}$, Mohamed Saafi $^{1 *}$, Jiawei Sun ${ }^{2}$, Yin Chi $^{1}$, Eric Whale ${ }^{3}$, David Hepworth $^{3}$, Jianqiao $\mathrm{Ye}^{1}$

${ }^{1}$ Department of Engineering, Lancaster University, Lancaster, LA1 4YW, UK

${ }^{2}$ Shaanxi Key Laboratory of Safety and Durability of Concrete Structures, Xijing University, Xi'an, China

${ }^{3}$ Cellucomp Ltd, Burntisland, Fife, KY3 9DW, UK

*Corresponding author. Tel. 44 (0) 1524594070

Email: m.saafi@lancaster.ac.uk (M.Saafi)

\section{Abstract}

In this paper, we show for the first time that environmentally friendly nanoplatelets synthesized from sugar beetroot waste with surface area and hydroxyl functional groups similar to those of graphene oxide (GO) can be used to significantly enhance the performance of cementitious composites. A comprehensive experimental and numerical simulation study was carried out to examine the performance of the bio waste-derived 2D nanoplatelets (BNP) in cementitious composites. The experimental results revealed that the addition of BNPs decreased the workability of the cement pastes due to their high surface area and dominant hydrophilic functional groups. The experimental results also revealed that the BNP sheets altered the morphology of the hydration phases of the cementitious composites. At 0.20-wt\%, the BNP sheets increased the content of the C-S-H gels. At higher concentrations (i.e., 0.40-wt\% and 0.60-wt\%), however, the BNP sheets increased the content of the calcium hydroxide $\left(\mathrm{Ca}(\mathrm{OH})_{2}\right)$ products and altered their sizes and morphologies.

The flexural results demonstrated that the 0.20-wt\% BNPs produced the highest flexural strength and modulus elasticity and they were increased by $75 \%$ and $200 \%$, respectively. The numerical simulations were in good agreement with the fracture test results. Both results showed that the 0.20-wt\% BNPs optimal concentration significantly enhanced the fracture properties of the 
cementitious composite and produced mixed mode crack propagation as a failure mode compared

33 to Mode I crack propagation for the plain cementitious composite due to combined crack bridging

34 and crack deflection toughening mechanisms. Because of this, the fracture energy and the fracture

35 toughness were increased by about $88 \%$ and $106 \%$, respectively.

\section{Introduction}

A great deal of research efforts has been devoted to improving the performance of cementitious composites using different nanoscale additives. Such additives offer tremendous promise for a wide range of uses in cementitious materials that could result in sustainable and high performance concrete structures with intelligence and multifunctional capabilities [1, 2]. For example, 41 cementitious composites incorporating reactive nanoparticles such as nano- $\mathrm{SiO}_{2}[3,4]$, nano- $\mathrm{TiO}_{2}$ $[5,6]$ and nano- $\mathrm{CaCO}_{3}[7,8]$ were found to exhibit improved mechanical properties and durability characteristics. This is because the high specific area of nanoparticles accelerates the hydration of cement, resulting in more Calcium Silicate Hydrate (C-S-H) gels. Furthermore, due to their small particle size, the nanoparticles tend to act as fillers, which results in a denser microstructure. However, these reactive nanoparticles tend to agglomerate at high concentrations and, due to their low aspect ratios, they cannot arrest the propagation of cracks, thereby are unable to enhance the

48 fracture properties of cementitious composites [9].

A significant body of research has demonstrated the benefits of adding carbonaceous nano50 additives such as carbon nanotubes (CNTs), carbon nanofibers (NFs) and graphene oxide (GO) to 51 cementitious materials. However, CNTs and NFs were shown to provide limited improvements 52 in the mechanical properties due to their agglomeration and lack of chemical and mechanical 53 bonding with the cement composite matrix [10,11]. The two dimensional (2D) GO is being 54 considered as an ideal candidate for reinforcing cementitious composites due to its distinctive 
properties such as large specific area, excellent mechanical properties and high dispersibility in water due to hydroxyl functional groups on its surface [12]. Studies reported that GO accelerates the hydration of cement and, regulate the growth and morphology of the hydration phases, leading to improvement in the mechanical properties of GO composites [13-15]. Studies also reported that GO influences pore volume distribution in cementitious materials [16]. It was shown that GO reduces the capillary pores and fills the micropores in the cement matrix [17]. Because of its large specific area, GO was found to bridge microcracks, thereby enhancing the stiffness and the fracture resistance of the cement matrix [18]. However, large-scale production of nano- $\mathrm{SiO}_{2}$ nano- $\mathrm{TiO}_{2}$, nano- $\mathrm{CaCO}_{3}, \mathrm{CNTs}$, NFs and GO and their applications in cementitious materials have been hampered by the high costs, un-scalability, complex manufacturing processes and, environmental, health and safety risk issues.

In this paper, we investigate for the first time the performance of cementitious composites containing novel and environmentally friendly low-cost 2D BNP sheets. The BNP sheets were produced from renewable materials such as sugar beetroot waste and resemble GO in terms of large specific area, excellent mechanical properties and hydroxyl functional groups with excellent dispersibility in water. The effect of different BNP concentrations on the workability, hydration phases, microstructure and mechanical properties was examined. The cracking behavior and the failure mode of the BNP cementitious composites were also examined and validated using numerical modelling.

\section{Experimental Program}

\subsection{Preparation of BNPs}

The BNP sheets were produced and supplied by our industrial partner Cellucomp Ltd, UK. The BNP sheets were synthesized from sugar beetroot waste recovered from existing industrial 
processes. The isolation of BNPs from sugar beetroot pulp is detailed in [19]. In summary, this

79 process involves alkali treatment of recovered sugar beetroot pulp with $0.5 \mathrm{M}$ of potassium hydroxide $(\mathrm{KOH})$ to extract the hemicellulose and pectin from the cells. The resulting mixture

81 was heated to $90^{\circ} \mathrm{C}$ for 5 hours and homogenized for 1 hour with a rotating mixer at rates between 11 and $30 \mathrm{~m} / \mathrm{s}$. This homogenization process separates the cells along the line of the middle lamella and breaks the separated cells into BNP sheets with about. The mixture was then filtered to remove

84 the dissolved materials. Finally, a nonionic surfactant (Span ${ }^{\mathrm{TM}}$ from Croda PLC, UK) was added to the BNP paste to coat the surface of the platelets to reduce aggregation thereby allowing BNPs

86 to be readily dispersed in aqueous solutions [19].

\subsection{Preparation of BNP cement pastes}

Portland cement (OPC) type CEM I 52.5N was used to prepare the cementitious composites with a water-to-cement ratio of 0.35. Commercially available superplasticizer (Glenium 51) was used at a concentration of $1-w t \%$ to enhance the workability of the cement pastes. The cement pastes were modified with BNPs at concentrations of 0.20, 0.40 and $0.60-w t \%$. The BNP was used asreceived and consisted of a paste with $4 \%$ solid and $96 \%$ water. The as-received BNPs were first added to the required water and superplasticizer, followed by mild sonication for 30 min using a probe sonicator. The resulting suspension was then blended with the cement and mixed for 7 min. For each BNP loading, 24 prisms (40 mm× $40 \mathrm{~mm} \times 160 \mathrm{~mm}$ ) were prepared to determine the mechanical and fracture properties of the cementitious composites. The prisms were demolded after 24 hrs then left to cure in water at a temperature of $21^{\circ} \mathrm{C}$ for 7,14 and 28 days.

\subsection{Characterization of BNPs}

99 Optical microscopy and ultraviolet-visible spectrophotometer were employed to examine the 
101 electron microscopy (SEM) (JSM-7800F) fitted with X-ray Energy Dispersive Spectrometer 102 (EDS) and X-ray diffraction (XRD) were used to determine the chemical composition, 103 morphology and microstructure of the BNP sheets. The EDS consisted of a X-max50 silicon drift 104 detector with an area of $50 \mathrm{~mm}^{2}$. The elemental analysis was conducted at a voltage of $10 \mathrm{kV}$ 105 under ambient temperature. The XRD system consisted of Rigaku SmartLab equipped with a Cu 106 rotating anode operating at $45 \mathrm{kV}$ and $200 \mathrm{~mA}$, a $\mathrm{Ge}(220)$ double bounce monochromator and a 107 Dtex-250 1d detector. The samples were analyzed with $\theta / 2 \theta$ scans with a rate 3 deg./min, under 108 ambient conditions. An Agilent Technologies Exoscan 4100 Fourier transform mid-infrared 109 spectrometer (FTIR) with diffuse sample interface was used to collect infrared diffuse spectra in 110 the range of $500-5000 \mathrm{~cm}^{-1}$. The instrumental conditions for spectral collection were 128 scans at

111 a resolution of $8 \mathrm{~cm}^{-1}$ under ambient conditions. The spectral changes both in terms of size and 112 position were used to identify the processes and chemical changes in the BNP sheets. 113 Thermogravimetric analysis (TGA) was carried to study the thermal stability of BNPs under 114 temperatures between 25 and $1100^{\circ} \mathrm{C}$ at a rate of $10{ }^{\circ} \mathrm{C} / \mathrm{min}$ in nitrogen (N2).

\section{2.4. Measurement of workability}

116 The effect of the BNP sheets on the workability of the cement pastes was assessed using a mini117 slump cone with a top diameter of $70 \mathrm{~mm}$, a bottom dimeter of $100 \mathrm{~mm}$ and a height of $60 \mathrm{~mm}$. 118 For each BNP concentration, the mini-slump diameter was measured according to [20]. The 119 average mini-slump diameter was based on three measurements.

\section{2.5. Characterization of hydration and microstructure BNP cementitious composites}

121 Samples were collected from the fractured flexural prisms at 7, 14 and 28 days to examine the 122 effect of BNP concentration on the hydration and microstructure of the cementitious composites. 123 TGA measurements were performed to estimate the degree of hydration (DOH) and the content of 
$124 \mathrm{Ca}(\mathrm{OH})_{2}$. In this experiment, the samples were heated from 25 to $1100{ }^{\circ} \mathrm{C}$ at a rate of $10{ }^{\circ} \mathrm{C} / \mathrm{min}$ 125 under nitrogen (N2). In addition, TGA measurements were performed on BNPs and cement 126 particles for correction purposes [21]. XRD analysis was carried out to further investigate the

$127 \mathrm{DOH}$ and determine the crystallinity of the hydration phases. SEM was employed to determine 128 the microstructure characteristics of the BNP-cementitious such as distribution of BNPs and crack 129 bridging mechanism. Transition electron microscopy (TEM) analysis was also conducted to study 130 the microstructure alteration processes associated with the addition of BNPs.

\subsection{Mechanical and fracture characterization of BNP cement composites}

132 As shown in Figs. 1a and 2a, four-point bending tests were conducted on 48 cementitious composite prisms $(40 \mathrm{~mm} \times 40 \mathrm{~mm} \times 160 \mathrm{~mm})(12$ prisms per BNP concentration $)$ under

134 displacement control with a rate of $0.1 \mathrm{~mm} / \mathrm{min}$. The flexural strength and modulus of elasticity 135 of the prisms were determined. Additionally, 48 cementitious prisms $(40 \mathrm{~mm} \times 40 \mathrm{~mm} \times 160 \mathrm{~mm})$ 136 equipped with a notch (3 $\mathrm{mm} \times 16 \mathrm{~mm}$ ) at the mid-span were subjected to a three-point bending test to evaluate the effect of BNPs on the fracture resistance of the prisms (Fig. 1b). The threepoint bending tests were also carried out under displacement control with a rate of $0.03 \mathrm{~mm} / \mathrm{min}$.

139 The crack mouth opening displacement (CMOD) was measured with a video gauge ${ }^{\mathrm{TM}}$ acquired

140 from Imetrum LTd. The video gauge system consisted of two lenses, an iMetrum controller and 141 a data acquisition system. As can be seen in Fig. 2b, five lines of 6 black dots with a dot diameter 142 of $4 \mathrm{~mm}$ and a center-to- center spacing of $5 \mathrm{~mm}$ were printed on the surface of the prisms around 143 the notch to define the region where the displacement is measured. The lenses were placed $1.5 \mathrm{~m}$ 144 away from the surface of the prisms (Fig. 2c). During testing, the positions of the dots were 145 continuously monitored by the lenses (Fig. 2c), and recorded along with the load. Both load and 146 positions were recorded at a frequency of $15 \mathrm{~Hz}$. The CMOD was obtained by monitoring the 
147 horizontal displacement between the two dots adjacent to the mouth of the crack as shown in Fig.

148 1b. The load vs CMOD, and the calculated fracture energy and fracture toughness were employed

149 to quantify the contribution of BNP to the fracture resistance of the cementitious composites.

$150 \quad$ 3. Results and discussion

151 3.1. Characterization of BNP sheets

152 The chemical components of the BNP sheets obtained from the EDX elemental analysis are given

153 in Table 1. As indicated in this table, the BNPs sheets contain mostly carbon, oxygen and

154 hydrogen. The main chemical components are carbon $47.61 \%$ and oxygen $46.91 \%$. The BNP

155 sheets contain some sodium and chloride impurities as a result of their chemical treatments. The

156 XRD pattern of the BNP sheets is shown in Fig. 3. As can be seen, the sheets exhibit two main

157 peaks at $2 \theta=15$ and 22, which represents the structure of cellulose. The XRD pattern suggests

158 that the structure of the BNP sheets can be divided into two regions. The narrow peak at $2 \theta=15$

159 represents the crystalline region of BNPs with a surface (110) plane. This surface (110) plane is

160 hydrophilic in nature due to the exposure to a large number of hydroxyl $(\mathrm{OH})$ groups, thus, has a

161 good dispersion in water [22]. The somewhat broad peak at $2 \theta=22.5$ with surface (200) plane

162 indicates the presence of crystalline and amorphous regions of BNPs. The amorphous region is

163 associated with the amorphous lignin and hemicellulose components of BNPs. The crystalline

164 region is highly hydrophobic because of the existence of $\mathrm{C}-\mathrm{H}$ moieties [22]. The crystallinity

165 index (CI) of BNPs was calculated using the following equation [22]:

166

$$
\mathrm{CI}(\%)=100 \times \frac{\mathrm{I}_{002}-\mathrm{I}_{\mathrm{am}}}{\mathrm{I}_{002}}
$$

167 where $I_{002}$ is the intensity of the XRD peak at $2 \theta=22.5$ and plane (200); $I_{a m}$ is the intensity of the 168 amorphous cellulose between the planes (200) and (110) at $2 \theta=18$. The calculated average $C I$ 
169

170

171

172

173

174

175

176

177

178

179

180

181

182

183

184

185

186

187

188

189

190

191

was about $64 \%$ which indicates high tensile strength and stiffness of the BNP sheets [22]. As a result, the proposed BNP sheets are good candidate materials for reinforcing cementitious composites. The FTIR spectrum of BNPs shown in Fig. 4 is similar to that reported by Li et al. [21]. This figure shows that the absorption in the $3600 \mathrm{~cm}^{-1}-3000 \mathrm{~cm}^{-1}$ region is the result of the vibrational stretching bands of hydrogen bonded hydroxyl groups which indicates the hydrophilic nature of the BNP sheets [22]. The pronounced peak at $2900 \mathrm{~cm}^{-1}$ is attributed to the stretching vibration of saturated $\mathrm{C}-\mathrm{H}$ in cellulose [22] and the peak at $1030 \mathrm{~cm}^{-1}$ is associated with the bending vibration of the absorbed water molecules [22]. Figure 4 shows that asymmetric and symmetric bending vibration bands exist at $1371 \mathrm{~cm}^{-1}$ and $1443 \mathrm{~cm}^{-1}$ [22].

The TGA/DTA results shown in Fig. 5 illustrate the thermal stability of BNPs. As depicted, the BNP sheets exhibit a small mass loss when heated from 25 to $200^{\circ} \mathrm{C}$ due to the evaporation of water content. A significant mass loss is observed between 200 and $700{ }^{\circ} \mathrm{C}$ as a consequence of elimination of hydroxyl groups and decomposition of the carbon chains [21]. The mass loss remains constant at temperatures between 700 and $1100^{\circ} \mathrm{C}$. The DTA spectrum shows a sharp peak at a temperature of about $260^{\circ} \mathrm{C}$ due to the dehydration of BNP sheets and a broad peak at about $500^{\circ} \mathrm{C}$ due to the decomposition of the BNP sheets. Overall, the BNP sheets exhibit a good thermal stability in the temperature range of 25 to $100^{\circ} \mathrm{C}$, which is the range in which cementitious composites are typically operating.

Figure 6 shows a typical micrograph of the BNP sheets, which indicates that the BNP sheets have a wrinkled texture resulting from the treatment of the sugar beetroot. This texture typically consists of crumpled and, stacked and overlapped thin sheets. This morphology enables the BNP sheets to interact mechanically with cement matrix, thus significantly enhancing the overall mechanical properties of the BNP composites [17]. It can be seen from Fig. 6 that the BNP sheets are 
192 composed of randomly oriented staked nanofibers with a diameter of about $10 \mathrm{~nm}$. It worth

193 mentioning that it was not possible to measure the dimensions of the BNP sheets, however,

194 according to the supplier, the average diameter of the flakes is about $50 \mu \mathrm{m}$.

\section{3.2. Colloidal properties and stability of BNP aqueous solutions}

196 The colloidal properties and stability of the BNP aqueous suspensions were determined in terms 197 of state of aggregation and microscale dispersion in aqueous solutions. Figure 7b depicts a typical 198 optical microscope image of the prepared BNP aqueous suspensions shown in Fig. 7a. As can be 199 seen, the BNP sheets seem to be uniformly dispersed without agglomeration. Figure 8 shows the 200 UV-vis spectroscopy spectra of the BNP aqueous solution as a function of sonication time. As 201 shown, the absorbance of the BNP sheets exhibits a maximum between 300 and $320 \mathrm{~nm}$ at all 202 sonication times. As the sonication time increases, the area under the spectrum lines representing 203 the absorbance increases as well, resulting in highly dispersed BNP sheets in water. It is worth 204 noting that the optical microscope image and absorbance spectra remained unchanged after 1 year, 205 thus indicating good stability of the BNP aqueous solutions.

\subsection{Influence of BNP on the workability of cement pastes}

207 Figure 9 shows the effect of the BNP sheets on the workability of the cement pastes. As can be 208 seen, the addition of 0.20 -wt\% BNPs did not affect the slab diameter of the cement paste, however, 209 the addition of 0.40-wt\% BNPs and 0.60-wt\% BNPs leads to a reduction in the slab diameter of 210 approximately $80 \%$ and $90 \%$, respectively, compared to the control mix. The slump decreases as

211 the content of BNPs increases, which indicates that the slump loss is proportional to the content of 212 BNPs. This is attributed to the high hydrophilicity and large specific area of the BNP sheets, 213 thereby requiring extra water to wet their surface. This reduces the free water content in the cement 214 pastes thereby reducing their workability. This is consistent with previous studies on cementitious 
215

216

217

218

219

220

221

222

223

224

225

226

227

228

229

230

231

232

233

234 for evaporation [24].

235 The DOH is directly correlated to the amount of $\mathrm{Ca}(\mathrm{OH})_{2}$, which can be calculated with the method 236 introduced by Wang et al. [25]. In this method, the mass loss in the temperature range $400-500^{\circ} \mathrm{C}$ 236 introduced by Wang et al. [25]. In this method, the mass loss in the temperature range $400-500^{\circ} \mathrm{C}$

materials containing GO sheets [23]. The workability of the cement pastes can be tuned by adding water reducing admixtures to promote the electrostatic repulsions between the cement particles and the BNP sheets.

\subsection{Influence of BNPs on the degree of hydration of cementitious composites}

The TGA results in terms of weight loss and derivative of the weight loss (DTA) are presented in Fig. 10 for the cementitious composites. In this figure, the percentage of the weight loss gradually decreases as the temperature increases and the inflections in the DTA represent the decomposition of specific phases of the cement paste composites. The TGA/DTA provides insight into the chemical reaction mechanisms in cementitious materials during heating. During this test, it was observed that C-S-H and carboaluminate phases lose their bound water in the temperature range $180-300{ }^{\circ} \mathrm{C}$; the dehydroxylation of $\left.\mathrm{Ca}(\mathrm{OH})_{2}\right)$ takes place in the temperature range $430-480^{\circ} \mathrm{C}$ and the decarbonation of calcium carbonate $\left(\mathrm{CaCO}_{3}\right)$ occurs in the temperature range $600-780^{\circ} \mathrm{C}$. From Fig. 10, it can be observed that the mass loss of the cementitious composites decreases with increasing BNP concentration. This is due to the increase of the high density C-S-H content and the creation of new intercalated BNP/C-S-H nanocomposites with higher density. This is consistent with Rehman et al. [24] findings where they have shown that the decrease in the mass loss of GO cementitious composites is attributed to both the bonding of $\mathrm{C}-\mathrm{S}-\mathrm{H}$ with the GO sheets and the increase of the $\mathrm{C}-\mathrm{S}-\mathrm{H}$ content. In this case, the GO sheets tend to increase the amount of C-S-H product, thereby filling the pores in the matrix resulting in less amount of water available

The DOH is directy 
238 the $\mathrm{Ca}(\mathrm{OH})_{2}$ content as a function of BNP concentration at 7, 14 and 28 days. At 7 and 28 days, 239 the overall trend observed is that the amount of $\mathrm{Ca}(\mathrm{OH})_{2}$ increases with increasing BNP 240 concentration. However, it is interesting to note that this trend is not evident at 14 days and this 241 needs to be investigated further. Overall, Fig. 11 suggests that the addition of BNP sheets 242 accelerates the hydration of cement, which results in the production of higher $\mathrm{Ca}(\mathrm{OH})_{2}$ contents at 2437 and 28 days.

244 According Cao et al. [26], the DOH can be obtained by dividing the amount of the chemically 245 bound water (CBW) per unit gram of unhydrated cement by the CBW of fully hydrated cement 246 which is $0.23 \mathrm{~g}$. The CBW can be obtained by dividing the mass loss between 140 and $1100{ }^{\circ} \mathrm{C}$ by 247 the final mass [26]. Figure 12 shows the DOH as a function of BNP concentration at 7, 14 and 28 248 days. As can be seen from this figure, the results clearly show that the DOH increases with 249 increasing BPN concentration. For example, the DOH of the cement paste with 0.60-wt\% BNPs 250 is increased with respect to the plain cement paste by 6\%, 7\%,and 9\% at 7, 14 and 18 days, 251 respectively.

252 The improvement in the hydration of cement can be attributed to the effect of the BNP sheets on 253 the reaction of the cement particles with water. According to Cao et al. [26], hydrophilic additives 254 disperse well the cement particles during mixing thereby producing uniform distributions of the 255 cement particles which results in higher DOH. Furthermore, the hydrophilic BNP sheets tend to 256 store water molecules on their surface thus acting like internal water reservoirs thereby releasing 257 free water for further hydration. This additional hydration further increases the amount of $\mathrm{Ca}(\mathrm{OH})_{2}$ 258 at 7 and 28 days. In addition, based on Cao et al. [26] hypothesis, the BNP sheets embedded into 259 the high density C-S-H could act as water channels and transfer water from the pore solution to 260 the un-hydrated cement cores, thus fueling the hydration of the cement particles [26]. 
262 The XRD patterns of the cementitious composites at 7, 14 and 28 days are shown in Fig. 13. As

263 it can be seen from this figure, typical hydration phases such as ettringite, calcium hydroxide $264 \mathrm{Ca}(\mathrm{OH})_{2}$, tricalcium silicate $\left(\mathrm{C}_{3} \mathrm{~S}\right)$ and $\left(\mathrm{CaCO}_{3}\right)$ are identified in all cementitious composites of all 265 ages using the pattern fitting and Rietveld refinement techniques. This indicates that the addition of 266 BNP sheets does not alter the type and structure of the hydration products of the cementitious. The 267 C-S-H hydration phases are difficult to identify by XRD analysis due to the lack of crystallinity 268 and indefinite composition. As depicted, the intensity of $\mathrm{Ca}(\mathrm{OH})_{2}$ increases with increasing BNP 269 concentration at 7, 14 and 28 days. This means the addition of the BNP sheets promotes the 270 hydration of cements thereby increasing the amount of the hydration products, which is in line 271 with the TGA results. Previous research on GO cementitious composites also reported similar 272 findings [25]. Another way to quantify the extent of hydration of cement as a result of BNPs is to 273 examine the magnitude of the intensity peaks of the detected $C_{3} S$ phase. From Fig. 13, it appears 274 that the intensity peaks of $\mathrm{C}_{3} \mathrm{~S}$ decreases when the BNP sheets are present. This could be attributed 275 to the interaction of $\mathrm{C}_{3} \mathrm{~S}$ with $-\mathrm{OH}$ and -R-CH2- functional groups on the surface of the BNP 276 sheets. Phases such as $\mathrm{C}_{3} \mathrm{~S}$ tend to react with water molecules adsorbed on the surface of the BNP 277 sheets thereby increasing the amount of hydration products.

278 Monitoring of $\mathrm{Ca}(\mathrm{OH})_{2}$ crystal size could shed light on the effect of BNP on the growth of C-S-H 279 phases. The size of $\mathrm{Ca}(\mathrm{OH})_{2}$ was obtained from the XRD analysis using the Scherrer equation. 280 The size of $\mathrm{Ca}(\mathrm{OH})_{2}$ as a function of BNP concentration at 28 days is shown in Fig. 14. From this 281 figure, it can be observed that the addition of BNP significantly decreases the size of the $\mathrm{Ca}(\mathrm{OH})_{2}$ 282 particles. This can be attributed to the fact that the BNP sheets promote the growth of C-S-H, thus 283 less space available for $\mathrm{Ca}(\mathrm{OH})_{2}$ to grow in size. Zheng et al. [27] found that when GO is present 
284 in the matrix, the size of $\mathrm{Ca}(\mathrm{OH})_{2}$ becomes smaller and the content of C-S-H increases, resulting

285 in a dense structure. It is noteworthy that the addition of 0.40 -wt\% BNPs and 0.60 wt\% BNPs

286 leads to a $\mathrm{Ca}(\mathrm{OH})_{2}$ size higher than that at 0.2 wt BNPs. This could be due to the fact that the

287 BNP sheets tend to restack at higher concentrations which in return dampers the grow of C-S-H

288 and allows $\mathrm{Ca}(\mathrm{OH})_{2}$ to grow in size. Therefore, based on this observation, it appears that the

289 content of C-S-H reaches a maximum at 0.20-wt\% BNPs at 28 days.

290

291

292

293

294

295

296

297

298

299

300

301

302

303

304

305

306

\subsection{Influence of BNP on the microstructure of cementitious composites}

The microstructure of the cementitious composites at 7, 14 and 28 days is presented in Figs.15-17. As shown, the microstructure of the plain cementitious composite at 7 days (Fig. 15a) contains unreacted cement particles as well as $\mathrm{Ca}\left(\mathrm{OH}_{2}\right)$ cubes and seeds-like particles, presumably due to a low DOH. The addition of 0.20 -wt\% BNPs leads to highly dense structure with some $\mathrm{Ca}\left(\mathrm{OH}_{2}\right)$ particles embedded into the C-S-H gel (Fig. 15b). The hydration phase $\mathrm{Ca}\left(\mathrm{OH}_{2}\right)$ in the form of cubes and rods-like crystals is observed in the microstructure of the composite with 0.40 -wt\% BNPs (Fig. 15c). The cementitious composite with 0.60-wt\% BNPs however, is marked by a high content of $\mathrm{Ca}(\mathrm{OH})_{2}$ seeds like crystals (Fig. 15d). It is worth to note that it is challenging to identify the BNP sheets in the SEM images. This is because the hydration products such as ettringite, $\mathrm{Ca}(\mathrm{OH})_{2}$ and $\mathrm{C}-\mathrm{S}-\mathrm{H}$ grow on their surface thus making their morphology undistinguishable.

From Figure 16a, it can be seen that the microstructure of the plain cementitious composite at 14 days becomes somewhat porous and contains a relatively high content of $\mathrm{Ca}\left(\mathrm{OH}_{2}\right)$ seeds-like crystals, whereas the cementitious composite with 0.20-wt\% BNPs (Fig. 16b) remains dense and its C-S-H content seems to increase. The cementitious composite with 0.40-Wt\% BNPs exhibits a different morphology where the $\mathrm{Ca}(\mathrm{OH})_{2}$ seeds transformed into rods-like crystals and begin to 
grow out from the matrix (Fig. 16c). As can be seen from Fig. 16d, when the BNP concentration

308 increases from 0.4-wt\% BNPs to 0.6-wt\% BNPs, the main hydration phase of the cementitious

309 composite is $\mathrm{Ca}(\mathrm{OH})_{2}$ in the form of agglomerates.

310 At 28 days of curing, the plain cementitious composite is mainly composed of $\mathrm{Ca}(\mathrm{OH})_{2}$ in the form

311 of regular polyhedral shaped particles (17a). On the other hand, the cementitious composite with

312 0.20-wt\% BNPs shows a compact structure with some layers of stacked fabrics-like $\mathrm{Ca}(\mathrm{OH})_{2}$

313 crystals embedded into high density C-S-H gel (Figs. 17b-c). Most of these $\mathrm{Ca}(\mathrm{OH})_{2}$ fabrics

314 appear to grow in one-direction. This could be the result of the interaction of the uniformly

315 distributed BNP sheets with the cement particles. We hypothesized that when uniformly

316 distributed, the BNP sheets adsorb onto the surface of the cement particles through their -OH and

317 -R- $\mathrm{CH}_{2}$ - functional groups. These functional groups then react with $\mathrm{C}_{3} \mathrm{~S}$ and $\mathrm{C}_{2} \mathrm{~S}$ to form

318 nucleation and growth sites for the hydration phases. The phase $\mathrm{C}_{2} \mathrm{~S}$ is less soluble than $\mathrm{C}_{3} \mathrm{~S}$, thus

319 a slower hydration rate of $\mathrm{C}_{2} \mathrm{~S}$ at these growth sites. At lower BNP concentrations, this could

320 allow more time for the hydration phases to self-assemble into fabric like-crystals. At a

321 concentration of $0.4-w t \%$, the cementitious composite contains C-S-H and $\mathrm{Ca}(\mathrm{OH})_{2}$ as hydration

322 products. The $\mathrm{Ca}\left(\mathrm{OH}_{2}\right)$ particles are in the form of elliptical needles growing out from the matrix

323 in two directions (Fig. 17d). When the BNP concentration increases to 0.6-wt\% BNPs, $\mathrm{Ca}(\mathrm{OH})_{2}$

324 is the main hydration product and the needle particles become cauliflower-like crystals as shown

325 in Figs. 17e-f. The SEM investigations suggest that the BNP sheets have the ability to regulate

326 the crystallization and morphology of the hydration products, which depends on the BNP

327 concentration in the cement matrix. The SEM investigations also suggest that the 0.20 -wt\% BNP

328 is the optimal concentration for increasing the C-S-H content in the cementitious composites. 

346 as [17]: images.

The TEM images in Fig. 18 show the effect of BNP on the microstructure of the cementitious composites at 28 days. As shown, the microstructure of the plain cementitious composite mainly consists of $\mathrm{Ca}(\mathrm{OH})_{2}$ crystals with a low C-S-H content (Fig. 18a). The addition of 0.2-wt\% BNPs leads to higher C-S-H content, fewer $\mathrm{Ca}(\mathrm{OH})_{2}$ crystals and denser microstructure (Fig. 18b). The TEM image shown in Fig. 18c confirms the existence of fabric-like crystals in the cementitious composite with 0.2-wt\% BNPs. From Fig. 18d, the microstructure of the cementitious with 0.4wt\% BNPs contains $\mathrm{Ca}\left(\mathrm{OH}_{2}\right)$ crystals embedded into C-S-H gel. On the other hand, significant amount of $\mathrm{Ca}\left(\mathrm{OH}_{2}\right)$ crystals is observed in the cementitious composite with 0.6-wt\% BNPs (Fig. 18e). Because of the hydroxyl functional groups on their surface, the BNP sheets are covalently bonded to C-S-H and $\mathrm{Ca}(\mathrm{OH})_{2}$ particles, thus making it challenging to identify them in the TEM

The TEM results further confirm the TGA, XRD and SEM findings that the BNP sheets accelerate the hydration of cement thus increasing both $\mathrm{C}-\mathrm{S}-\mathrm{H}$ and $\mathrm{Ca}(\mathrm{OH})_{2}$ contents in the cementitious composites. The TEM results also confirm that the 0.20 -wt $\%$ BNPs concentration produces the highest C-S-H content.

\subsection{Influence of BNP on the Mechanical properties of cementitious composites}

The flexural strength $\left(\sigma_{c}\right)$ and the modulus of elasticity $\left(E_{c}\right)$ of the cement prisms were calculated

$$
\begin{aligned}
& \sigma_{c}=\frac{3 P a}{b^{3}} \\
& E_{C}=\frac{a m\left(3 l^{2}-4 a^{2}\right)}{4 b^{4}}
\end{aligned}
$$

where $l$ is the length of the prism between the supports, $a$ is the distance between the support and the loading point, $P$ is the maximum applied load, $b$ is the width and thickness of the prism and, $m$ is the slope of the tangent to the straight-line portion of the load-deflection curve. 
352 The effect of BNP concentration on the average flexural strength of the cementitious composites

353 at 7, 14 and 28 days is given in Fig. 19. This figure shows that at 7 and 14 days, the addition of

354 0.20-wt\% BNPs increases the flexural strength of the cementitious composites by $23 \%$ and $20 \%$

355 respectively whereas, no significant increase is observed at 0.4-wt\% BNPs and 0.6-wt\% BNPs

356 concentrations. At 28 days, the flexural strength is increased by about $75 \%$ and $50 \%$ at BNP

357 concentrations of $0.20-w t \%$ and $0.40-w t \%$, respectively. This increase however, significantly

358 diminishes at 0.60-wt\% BNPs.

359 Figure 20 presents the average modulus of elasticity of the cementitious composites as a function 360 of BNP concentration at 28 days. As depicted, the modulus of elasticity follows a similar trend to 361 that of the flexural strength at 28 days. A significant modulus of elasticity gain of about $200 \%$ is 362 achieved at BNP concentration of 0.20-wt\%. However, like the flexural strength, the modulus of 363 elasticity diminishes at higher BNP concentrations.

364 The improvement of mechanical properties of the cementitious composites at BNP concentrations 365 of $0.20-w t \%$ and $0.40-w t \%$ can be attributed to higher C-S-H content and better packing density. 366 The diminishing of these mechanical properties at BNP concentrations higher than 0.20 -wt\% is 367 probably because of the synergetic effect of the restacking of the BNP sheets and the high content 368 of $\mathrm{Ca}\left(\mathrm{OH}_{2}\right)$ crystals. We hypothesize that at higher BNP concentrations, severe restacking of the 369 BNP sheets occurs in the high alkaline cement pastes, thereby reducing their mechanical 370 properties. The high alkaline cement pore solution rich in $\mathrm{Ca}^{2+}$ ions attenuates the hydroxyl groups 371 on the surface of the BNP sheets and high van-der-Waals forces are created, thereby allowing the 372 sheets to stack on top of each other to form stiff agglomerates [17]. These agglomerates weaken 373 the matrix, causing the cement composites to fail in a brittle manner with lower mechanical 374 properties. The high content of $\mathrm{Ca}(\mathrm{OH})_{2}$ crystals in the cementitious composites containing 0.40- 
375 wt\% BNPs and 0.60-wt\% BNPs could also diminish the mechanical properties. $\mathrm{Ca}(\mathrm{OH})_{2}$ crystals

376 are typically brittle in nature thereby weakening the cement matrix by making it highly susceptible

377 to a brittle fracture. Based on this, 0.20-wt\% BNPs is the optimal content for maximum

378 mechanical properties.

\subsection{Interaction mechanism between BNP and cement matrix}

380 The improvement of the mechanical properties of the cementitious composites containing 0.20381 wt\% BNPs and 0.40-wt\% BNPs can be attributed both to improved hydration kinetics of the 382 cement particles which results in higher C-S-H content and the reinforcing effect of the BNP 383 sheets. The reinforcing effect is controlled by the mechanical interaction between the BNP sheets 384 and the cementitious matrix coupled with chemical cross-linking type bonding. As showing in 385 Fig. 6b, the BNP sheets are characterized by a wrinkled texture. This morphology plays a 386 significant role in the toughening and load transfer mechanisms in the cementitious composites, 387 because it enhances the mechanical interlocking [17]. Saafi et al. [17] have shown that the 388 mechanical interaction between the BNP sheets and the cement matrix can be examined using both 389 the morphology of the BNP sheets and the shear lag model. The strain $\varepsilon_{\mathrm{p}}$ in the BNP sheet as a 390 function of the strain $\varepsilon_{\mathrm{m}}$ in the cement matrix is [28]:

$$
\varepsilon_{p}=\varepsilon_{m}\left[1-\frac{\cosh \left(n s \frac{x}{l}\right)}{\cosh \left(\frac{n s}{l}\right)}\right]
$$

$$
n=\sqrt{\frac{2 G_{m}}{E_{p}} \frac{t}{T}}
$$

393 where $s$ is the aspect ratio of the BNP sheet $(1 / t), n$ represents the interfacial stress transfer

394 efficiency and the product ns represents both the morphology of the BNP sheet and the degree of 
395 interaction with the cementitious material. $G_{m}$ is the shear modulus of the cementitious material, $396 t$ is the thickness of the BNP sheet, $T$ is the total thickness of the cementitious material, $E_{p}$ is the 397 modulus elasticity of the BNP sheet and $l$ is the length of the BNP sheet in the $\mathrm{x}$ direction [28].

398 The mechanical interaction between the two materials is slowly depending on the morphology of 399 the BNP sheet (Fig. 6b) which can be characterized by the wavelength $\lambda$ and the amplitude A of 400 the wrinkles and ribs as [29]:

$$
\lambda^{4}=\frac{4 \pi^{2} v(t l)^{2}}{3\left(1-v^{2}\right) \varepsilon}
$$

$$
A^{2}=\left[\frac{16 \varepsilon v}{3 \pi^{2}\left(1-v^{2}\right)}\right]^{\frac{1}{2}} t l
$$

where $\varepsilon$ is the compressive strain in the BNP sheet resulting from the chemical treatment, $v$ is the Poisson's ratio of the BNP sheet. The shear stress $\tau$ between the BNP sheet and the matrix in the 405 direction $x$ is [29]:

$$
\tau=n E_{p} \varepsilon_{m} \frac{\sinh \left(n s \frac{x}{l}\right)}{\cosh \left(\frac{n s}{2}\right)}
$$

407 Equations (4), (5), (6) and (7) indicate that the observed increase in the mechanical properties of 408 the BNP cementitious composites at 0.20-wt\% BNPs and 0.40-wt\% BNPs is in part as a result of 409 a good mechanical interaction between the two materials which is controlled by properties of the 410 BNP sheets mainly the high aspect ratio, the modulus of elasticity and the surface morphology.

411 The BNP characterization results indicate that the BNP sheets are fully decorated with hydroxyl 412 functional groups which are responsible for their high chemical reactivity. These functional 413 groups are believed to adsorb on the surface of the cement particles. In this case, the main chemical 414 components of the cement $\mathrm{C}_{2} \mathrm{~S}$ and $\mathrm{C}_{3} \mathrm{~S}$ hydrate over the surface of the BNP sheets, leading to 
415 intercalated C-S-H/BNP and $\mathrm{Ca}(\mathrm{OH})_{2} / \mathrm{BNP}$ particles. Figure 21 shows the chemical interaction

416 of the BNP sheets with C-S-H at 28 days. The strong interfacial covalent bonding between C-S-

$417 \mathrm{H}$ and the functionalized BNP sheets enhances the stress transfer thus improving the overall

418 mechanical properties of the cementitious composites at BNP concentrations of 0.20 -wt $\%$ and $419 \quad 0.40-w t \%$.

420 As shown in Figs 22 and 23, the maximum percentage increase in the flexural strength of the BNP421 cementitious composite at 0.20 -wt $\%$ is compared with that of several cementitious composites 422 containing MWCNT and GO obtained from [30-47]. As shown in these figures, overall, the BNP 423 outperforms MWCNTs and GO. This is due to high dispersibility of the BNP flakes in the cement 424 paste. In addition, the BNP flakes contains more hydroxyl groups, thus better chemical reactivity 425 which improves the hydration of cement.

3.9. Influence of BNP on the Fracture properties of cementitious composites: numerical simulation

427 and experimental results

428 The three-point-bending fracture tests were numerically simulated by ABAQUS to further 429 elucidate their fracture behavior and validate the experimental results. In order to capture the 430 evolution of cracks, extended finite element method (X-FEM) was used as the finite element mesh 431 need not conform to the varying internal boundaries caused by the propagation of cracks. Hence, 432 a single mesh is sufficient for completing entire simulation of a test. Fig.24 shows the geometry 433 of the X-FEM model for the cement prism with and without BNP. The dimensions of the prisms 434 are identical to the ones used in the laboratory tests. The prisms are simply supported near the two 435 ends. The force is applied at the mid-span, where a $16 \mathrm{~mm}$ long virtual crack perpendicular to the 436 bottom surface is embedded. The prisms were discretized by cubic C3D8R elements. Mesh 437 sensitivity analysis was carried out and it was found that elements of $2 \mathrm{~mm} \times 2 \mathrm{~mm} \times 2 \mathrm{~mm}$ were 
sufficiently small to achieve converged results. The maximum principal stress fracture criteria was used to initiate fracture. The material properties of the cementitious composites of the XFEM models were taken from the laboratory tests described in Sections 3.7 and 3.8 and, the details are shown in Table 2.

Figure 25 shows the predicted and measured load vs CMOD response plain prisms and prisms with 0.20 -wt\% BNPs (optimal BNP concentration). It is worth mentioning that it was not possible to experimentally capture the post-cracking response of the prisms due to the limitation of the test machine employed in this investigation. As shown, at the optimal BNP concentration, the fracture load, stiffness and the amount of absorbed energy are significantly increased, thus better fracture properties. Figure 25 shows that the FEM model provides a good prediction of the fracture load of the prisms, though it overestimates their stiffness.

The experimental fracture energy $G_{f}$ and the fracture toughness $K_{I C}$ were calculated according to [48]. The calculated average $G_{f}$ and $K_{I C}$ are about $12.55 \mathrm{Nm} / \mathrm{m}^{2}, 0.16 \mathrm{MPa} \mathrm{m}^{0.5}$, respectively, for the plain prism, and $23.61 \mathrm{Nm} / \mathrm{m}^{2}$ and $0.33 \mathrm{MPa} \mathrm{m}^{0.5}$ respectively, for the prism containing 0.20 wt\% BNPs. This shows that the addition of BNPs increases $G_{f}$ and $K_{I C}$ by about $88 \%$ and $106 \%$, respectively.

Figure 26 compares the observed and simulated cracking behavior of the prisms. As shown, there is a good agreement between both the simulated and experimental failure mode of the prisms. The failure mode of the plain cement prism is Mode I crack propagation along the surface of the initial notch. The failure mode of the prism containing 0.20-wt\% BNPs is somewhat mixed mode (Mode I+II) crack propagation in the form of an inclined crack initiated slightly above the notch tip. This can be attributed to the BNP crack deflection effect and the improved flexural strength. 
The significant increase in the fracture properties of the BNP cementitious composites is attributed

461 to the toughening mechanisms originated from the presence of BNP sheets. As shown in Fig. 27b,

462 the toughness seems to be governed by the crack-bridging mechanism where the BNP sheet

463 appears to disentangle into nanofibers under stress and bridge the crack. Figures 27c and 27d show

464 another toughening mechanism associated with mainly crack deflection that bypasses the BNP

465 sheet at the BNP/matrix interface.

466 Conclusions

467 In this paper, we demonstrate for the first time that nanoplatelets synthesized from food waste such as sugar beetroot can be used as a low-cost and renewable reinforcing material in cementitious materials. The proposed BNP sheets exhibit some characteristics of GO such as hydrophilic functional groups, high specific area and good dispersibility in water. An integrated experimental

471 and numerical simulation approach was employed to evaluate the performance of cementitious 472 composites containing BNP sheets.

473 Because of their high surface area and hydrophilicity, the BNP sheets tend to consume water 474 thereby reducing the workability of the cement pastes at BNP concentrations higher than 0.20$475 \mathrm{wt} \%$. The incorporation of the BNP sheets increased the degree of hydration of cement due to the 476 active participation of their functional groups and their supply of water molecules, resulting in 477 high content of the hydration products. The microstructure, the type of the hydration phases and 478 the mechanical properties seemed to be highly dependent on the amount of the BNP sheets. 479 Compared to the plain cementitious composite, the cementitious composite with 0.20-wt\% BNPs 480 showed a microstructure with better packing density with C-S-H as the main hydration phase. At 481 higher BNP concentrations, $\mathrm{Ca}(\mathrm{OH})_{2}$ was the main hydration phase. The BNP sheets appeared to 482 regulate the morphology of $\mathrm{Ca}(\mathrm{OH})_{2}$ crystals. At 28 days, the regular polyhedral shaped $\mathrm{Ca}(\mathrm{OH})_{2}$ 
483

484

485

486

487

488

489

490

491

492

493

494

495

496

497

498

499

500

501

502

503

504

505

particles in the plain cementitious composite changed to stacked fabric-like $\mathrm{Ca}(\mathrm{OH})_{2}$ at 0.20 -wt\% BNPs, elliptical needles at 0.40-wt\% BNPs and cauliflower-like $\mathrm{Ca}(\mathrm{OH})_{2}$ at 0.60 -wt\%.

The experimental results suggested that 0.20 -wt $\%$ BNPs is the optimal concentration for maximum amount of C-S-H gel and mechanical properties. At this BNP content, the flexural strength and the modulus of elasticity were by increased by $75 \%$ and $200 \%$, respectively due to a good packing density of the cement paste. However, these mechanical properties diminished at higher BNP concentrations due to restacking of BNP and increased amount of $\mathrm{Ca}(\mathrm{OH})_{2}$. Both experimental and numerical simulation results showed that the fracture resistance of the cementitious composites were significantly improved at 0.20-wt\% BNPs due to the crack bridging and crack deflection effects of the BNP sheets. At this concentration, the fracture energy and the fracture toughness were increased by about $88 \%$ and $106 \%$, respectively.

Although further studies are required to investigate the durability of the proposed cementitious composites and optimize their properties, the proposed 2D BNP has the potential to create durable and high performance cementitious materials with low-embodied carbon for various applications in the construction sector.

\section{Acknowledgements}

The authors would like to acknowledge the financial support H2020-MSCA-IF under the grant No. 799658_B-SMART: Biomaterials derived from food waste as a green route for the design of eco-friendly, smart and high performance cementitious composites for the next built infrastructure. The authors would like to thank the Iraqi Government for providing a PhD scholarship to the first author. The authors are grateful for Cellucomp Ltd for providing the BNP material. 


\section{References}

[1] Y. Reches, Nanoparticles as concrete additives: Review and perspectives, Construction and Building Materials 175 (2018) 483-495.

[2] F. Sanchez, K. Sobolev Nanotechnology in concrete-a review, Construction and building materials 397 24(11) (2010) 2060-2071.

[3] T. Ji, Preliminary study on the water permeability and microstructure of concrete incorporating nano-SiO 2. Cement and Concrete Research. 2005;35(10):1943-7.

[4] H. Li, H.G. Xiao, J. Yuan, J. Ou, Microstructure of cement mortar with nano-particles. Composites Part B: Engineering. 2004;35(2):185-9.

[5] H. Li, H. Xiao, X. Guan, Z. Wang, L. Yu, Chloride diffusion in concrete containing nano-TiO 2 under coupled effect of scouring. Composites Part B: Engineering. 2014;56:698-704.

[6] L. Senff, D. Tobaldi, S. Lucas, D. Hotza, V. Ferreira, J. Labrincha, Formulation of mortars with nano-SiO 2 and nano-TiO 2 for degradation of pollutants in buildings. Composites Part B: Engineering. 2013;44(1):40-7.

[7] S. Kawashima, JWT. Seo, D. Corr, MC. Hersam, SP. Shah, Dispersion of CaCO3 nanoparticles by sonication and surfactant treatment for application in fly ash-cement systems. Materials and structures. 2014;47(6):1011-23.

[8] W. Li, Z. Huang, T. Zu, C. Shi, WH. Duan, SP. Shah, Influence of nanolimestone on the hydration, mechanical strength, and autogenous shrinkage of ultrahigh-performance concrete. Journal of Materials in Civil Engineering. 2015;28(1):04015068.

[9] Z. Pan, L. He, L. Qiu, AH. Korayem, G. Li, JW. Zhu, et al., Mechanical properties and microstructure of a graphene oxide-cement composite. Cement and Concrete Composites. 2015; 58:140-7. 
529 [10] BM. Tyson, Abu Al-Rub RK, Yazdanbakhsh A, Grasley Z, Carbon nanotubes and carbon

530526 nanofibers for enhancing the mechanical properties of nanocomposite cementitious materials.

531 Journal of Materials in Civil Engineering. 2011;23(7):1028-35.

532 [11] A, Cwirzen, K. Habermehl-Cwirzen, A. Nasibulin, E. Kaupinen, P. Mudimela, V. Penttala, 533 SEM/AFM studies of cementitious binder modified by MWCNT and nano-sized Fe needles.

534 Materials Characterization. 2009;60 (7):735-40.

535 [12] Y. Xu, J. Zeng, W. Chen, R. Jin, B. Li, Z. Pan, A holistic review of cement composites 536 reinforced with graphene oxide, Construction and Building Materials 171 (2018) 291-302.

537 [13] S. Lv, Y. Ma, C. Qiu, T. Sun, J. Liu, Q. Zhou, Effect of graphene oxide nanosheets of 538 microstructure and mechanical properties of cement composites, Constr. Build. Mater. 49 (2013) 539 121-127. doi:10.1016/j.conbuildmat.2013.08.022.

540 [14] M.M. Mokhtar, S.A. Abo-El-Enein, M.Y. Hassaan, M.S. Morsy, M.H. Khalil, Mechanical 541 performance, pore structure and micro-structural characteristics of graphene oxide nano platelets 542 reinforced cement, Construction Building Materials 138 (2017) 333-339. 543 doi:10.1016/j.conbuildmat.2017.02.021.

544 [15] X. Li, A.H. Korayem, C. Li, Y. Liu, H. He, J.G. Sanjayan, W.H. Duan, Incorporation of 545 graphene oxide and silica fume into cement paste: A study of dispersion and compressive strength, 546 Construction and Building Materials 123 (2016) 327-335.

547 [16] K. Gong, Z. Pan, A.H. Korayem, L. Qiu, D. Li, F. Collins, C.M. Wang, W.H. Duan. 548 Reinforcing Effects of Graphene Oxide on Portland Cement Paste, J. Mater. Civ. Eng. 27 (2015) 549 A4014010. doi:10.1061/(ASCE)MT.1943-5533.0001125. 
550 [17] M. Saafi, L. Tang, J. Fung, M. Rahman, J. Liggat, Enhanced properties of graphene/fly ash

551 geopolymeric composite cement, Cem. Concr. Res. $67 \quad$ (2015) 292-299,

552 doi:10.1016/j.cemconres.2014.08.011.

553 [18] B. Wang, R. Jiang 1, Z. Wu, Investigation of the mechanical properties and microstructure of 554 graphene nanoplatelet-cement composite, Nanomaterials, 2016, 6 (11), 200; 555 doi:10.3390/nano6110200.

556 [19] USA Patent No: 9,834,664, Cellucomp Limited, Fife, UK.

557 [20] F. Collins, J. Lambert, W. H. Duan, The influences of admixtures on the dispersion, 558 workability, and strength of carbon nanotube-OPC paste mixtures, Cement \& Concrete 559 Composites, 2012, 34, 201-2017, doi: 10.1016/j.cemconcomp.2011.09.013.

560 [21] Y. Cao, P. Zavaterri, J. Youngblood, R. Moon, J. Weiss, The influence of cellulose 561 nanocrystal additions on the performance of cement paste, Cement \& Concrete Composites, 2015 562 ,56, 73-83, doi:10.1016/j.cemconcomp.2014.11.008.

563 [22] M. Lia, L. Wang, D. Lia, YL. Cheng, B. Adhikari, Preparation and characterization of 564 cellulose nanofibers fromde-pectinated sugar beet pulp, Carbohydrate Polymers 102 (2014) 136565 143, doi:10.1016/j.carbpol.2013.11.021.

566 [23] X. Li, YM. Liu, WG. Li, CY. Li, JG. Sanjayan, WH. Duan, Z. Li, Effects of graphene oxide 567 agglomerates on workability, hydration, microstructure and compressive strength of cement paste, 568 Construction and Building Materials 145 (2017) 402-410, doi: 569 10.1016/j.conbuildmat.2017.04.058.

570 [24] SK. Rehman, Z. Ibrahim, SA. Memon, MdT. Aunkor, MF. Javed, K. Mehmood, SM A. Shah, 571 Influence of graphene nanosheets on rheology,microstructure, strength development and self- 
572 sensing properties of cement based composites, Sustainability 2018, 10(3), 822; doi: 573 10.3390/su10030822.

574 [25] L. Wang, S. Zhang, D. Zheng, H. Yang, H. Cui, W. Tang, D. Li, Effect of graphene oxide 575 (GO) on the morphologyand microstructure of cement hydration products, Nanomaterials (Basel). 5762017 Dec 5;7(12). pii: E429. doi: 10.3390/nano7120429.

577 [26] Y. Cao, P. Zavaterri, J. Youngblood, R. Moon, J. Weiss, The influence of cellulose 578 nanocrystal additions on the performance of cement paste, Cement \& Concrete Composites 56 579 (2015) 73-83, doi: 10.1016/j.cemconcomp.2014.11.008.

580 [27] Q. Zheng, B. Han, X. Cui, X. Yu, J. Ou, Graphene-engineered cementitious composites: Small 581 makes a big impact, Nanomaterials and Nanotechnology, Volume 7 (2017): 1-18, doi: $582 \quad 10.1177 / 1847980417742304$.

583 [28] L. Gong, I.A. Kinloch, R.J. Young, I. Riaz, R. Jalil, K.S. Novoselov, Interfacial stress transfer 584 in a graphene monolayer nanocomposite, Adv. Mater. 22 (24) (2010), 2694-2697.

585 [29] L. Tapasztó, T. Dumitrica, S. Jin-Kim, P. Nemes-Incze, C. Hwang, L.P. Biró, Break-down of 586 continuummechanics for nanometer-wavelength rippling of graphene, Nat. Phys.8 (2012) 739$587 \quad 741$.

588 [30] Tyson, B. M., Abu Al-Rub, R. K., Yazdanbakhsh, A. \& Grasley, Z. 2011. Carbon Nanotubes 589 and Carbon Nanofibers for Enhancing the Mechanical Properties of Nanocomposite Cementitious 590 Materials.(Author Abstract)(Report). Journal of Materials in Civil Engineering, 23(7), 1028.

591 [31] Luo, J., Duan, Z. \& Li, H. 2009. The Influence of Surfactants on the Processing of Multi592 Walled Carbon Nanotubes in Reinforced Cement Matrix Composites. physica status solidi (a), 206 593 (12), 2783-2790. 
[32] Metaxa, Z. S., Konsta-Gdoutos, M. S. \& Shah, S. P. 2012a. Carbon Nanofiber Cementitious

595 Composites: Effect of Debulking Procedure on Dispersion and Reinforcing Efficiency. Cement 596 and Concrete Composites, 36(1).

597 [33] Xu, S., Liu, J. \& Li, Q. 2015. Mechanical Properties and Microstructure of Multi- Walled 598 Carbon Nanotube-Reinforced Cement Paste. Construction and Building Materials, 76, 16-23.

599 [34] Li, G., Wang, P, Zhao, X. 2005. Mechanical behavior and microstructure of cement 600 composites incorporating surface-treated multi-walled carbon nanotubes. Carbon. 43, 1239-1245.

601 [35] Cwirzen. A, Habermehl Cwirzen. K and Penttala. V "Surface decoration of carbon nanotubes 602 and mechanical properties of cement/carbon Nanotube composites" in Advances in Cement 603 Research, 2008, 20, No. 2, April, pp.65-73.

604 [36] Musso, S., Tulliani, J.-M., Ferro, G. \& Tagliaferro, A. 2009. Influence of Carbon Nanotubes 605 Structure on the Mechanical Behavior of Cement Composites. Composites Science and 606 Technology, 69(11), 1985-1990.

607 [37] Konsta-Gdoutos, M. S., Metaxa, Z. S. \& Shah, S. P. 2010. Highly Dispersed Carbon Nanotube 608 Reinforced Cement Based Materials. Cement and Concrete Research, 40(7), 1052-1059.

609 [38] Chan, L. Y. \& Andrawes, B. 2010. Finite Element Analysis of Carbon Nanotube/Cement 610 Composite with Degraded Bond Strength. Computational Materials Science, 47(4), 994-1004.

611 [39] Metaxa, Z. S., Seo, J.-W. T., Konsta-Gdoutos, M. S., Hersam, M. C. \& Shah, S. P. $2012 b$. 612 Highly Concentrated Carbon Nanotube Admixture for Nano-Fiber Reinforced Cementitious 613 Materials. Cement and Concrete Composites, 34(5), 612-617.

614 [40] Lv, S., Liu, J., Sun, T., Ma, Y. \& Zhou, Q. 2014a. Effect of Go Nanosheets on Shapes of 615 Cement Hydration Crystals and Their Formation Process. Construction and Building Materials, 616 64, 231-239. 
617 [41] Lv, S., Ting, S., Liu, J. \& Zhou, Q. 2014b. Use of Graphene Oxide Nanosheets to Regulate 618 the Microstructure of Hardened Cement Paste to Increase Its Strength and Toughness. 619 CrystEngComm, 16(36), 8508-8516.

620 [42] Lv, S., Ma, Y., Qiu, C., Sun, T., Liu, J. \& Zhou, Q. 2013. Effect of Graphene Oxide 621 Nanosheets of Microstructure and Mechanical Properties of Cement Composites. Construction and 622 Building Materials, 49, 121-127.

623 [43] Shang, Y., Zhang, D., Yang, C., Liu, Y. \& Liu, Y. 2015. Effect of Graphene Oxide on the 624 Rheological Properties of Cement Pastes. Construction And Building Materials, 96, 20-28.

625 [44] Li, X., Liu, Y. M., Li, W. G., Li, C. Y., Sanjayan, J. G., Duan, W. H. \& Li, Z. 2017. Effects 626 of Graphene Oxide Agglomerates on Workability, Hydration,

627 [45] Zhou, C., Li, F., Hu, J., Ren, M., Wei, J. \& Yu, Q. 2017. Enhanced Mechanical Properties of 628 Cement Paste by Hybrid Graphene Oxide/Carbon Nanotubes. Construction and Building 629 Materials, 134, 336-345.

630 [46] Muhit B. A., 2015 Investigation on the Mechanical, Microstructural, and Electrical Properties 631 of Graphene OxideCement Composite. Master thesis, University of Central Florida.

632 [47] Zhao, L., Guo, X., Liu, Y., Ge, C., Guo, L., Shu, X. \& Liu, J. 2017. Synergistic Effects of 633 Silica Nanoparticles/Polycarboxylate Superplasticizer Modified Graphene Oxide on Mechanical 634 Behavior and Hydration Process of Cement Composites. RSC Adv., 7(27), 16688-16702.

635 [48] Y. Hu, D. Luo, P. Li, Q. Li., G. Sun, Fracture toughness enhancement of cement paste with 636 multi-walled carbon nanotubes, Construction and Building Materials 70 (2014), 332-338. 

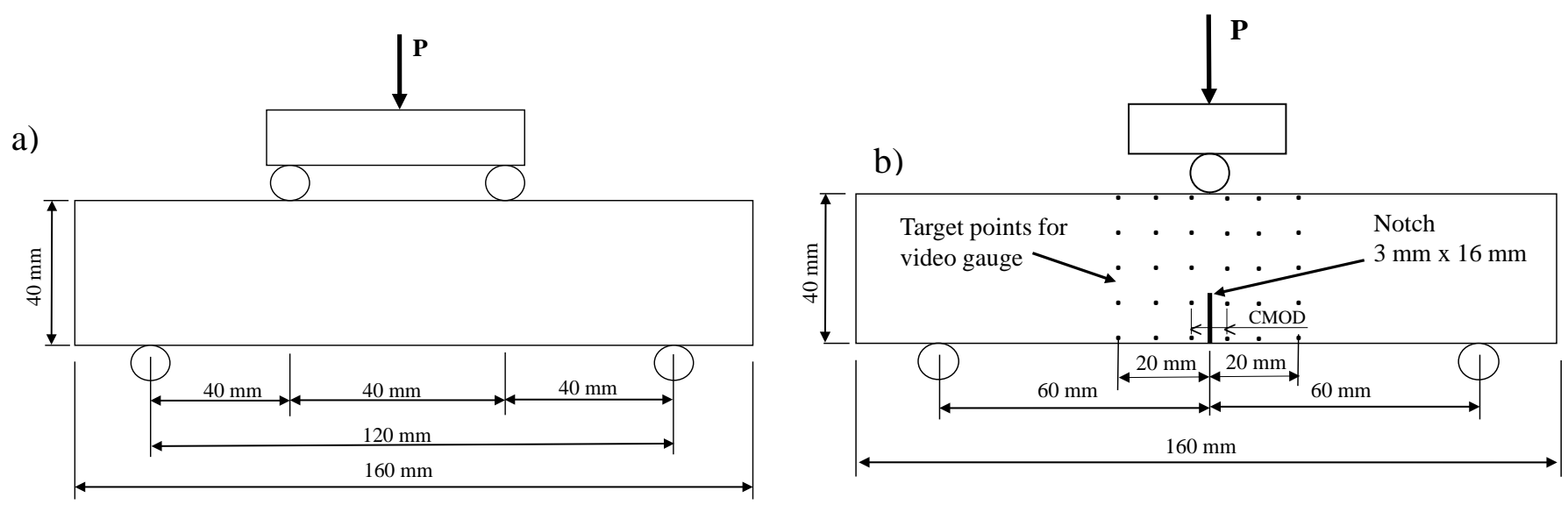

Fig. 1. Test details of the prisms and schematic of test setups. a) flexural strength test setup, b) fracture test setup (not to scale).

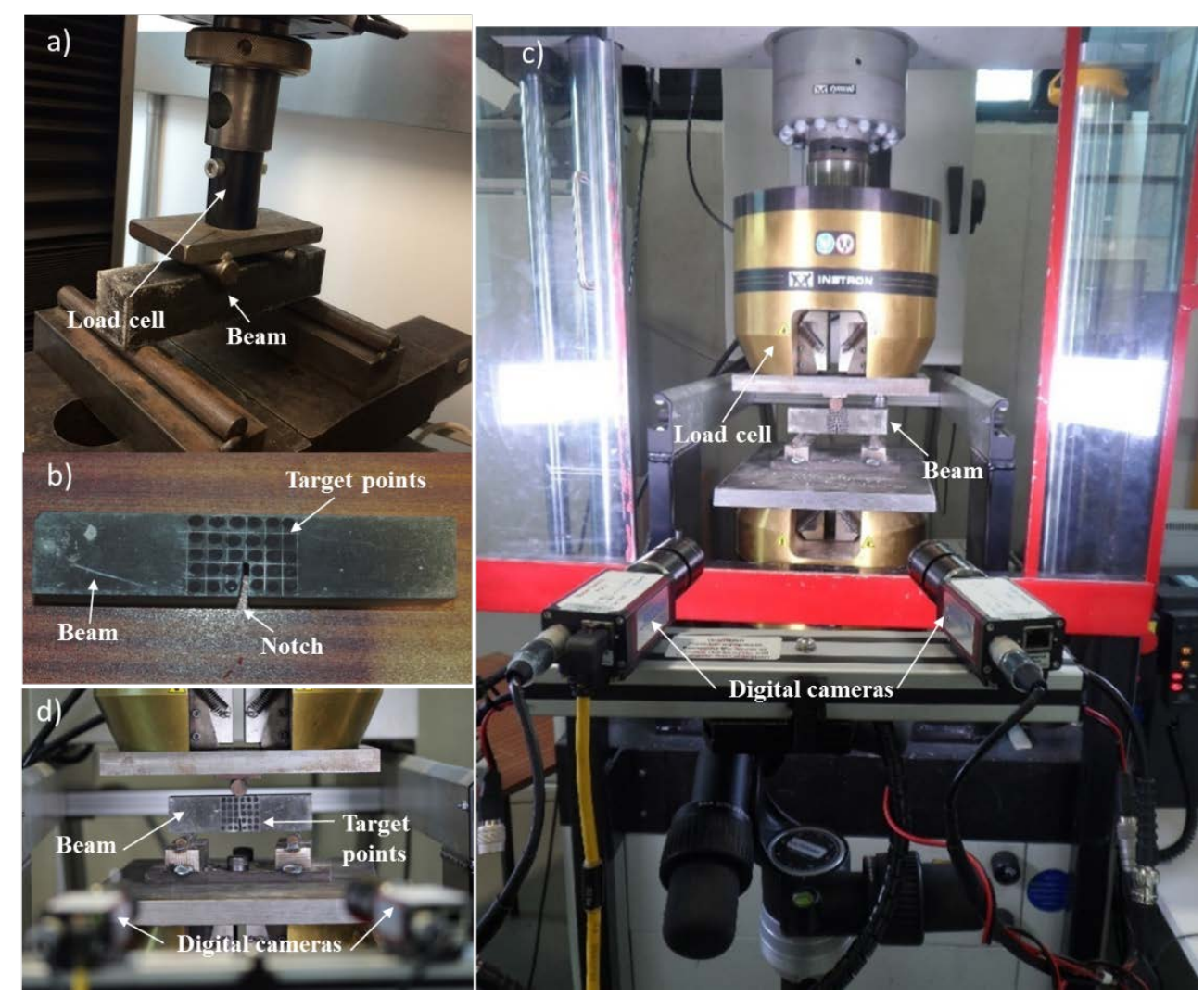

Fig. 2. Images of the experimental test setups. a) flexural strength test setup, b) notched prism with optical grid for fracture test., c) fracture test setup with digital camera for CMOD measurement, d) close up view of the notched prism under load.

Table 1: Chemicals composition of the BNP sheets

\begin{tabular}{lcccccccc}
\hline Chemical components & $\mathrm{C}$ & $\mathrm{O}$ & $\mathrm{Ca}$ & $\mathrm{Al}$ & $\mathrm{Cl}$ & $\mathrm{Mg}$ & $\mathrm{Si}$ & $\mathrm{Na}$ \\
\hline Content (\%) & 47.6 & 46.9 & 1.9 & 1.8 & 0.7 & 0.5 & 0.4 & 0.2 \\
\hline
\end{tabular}




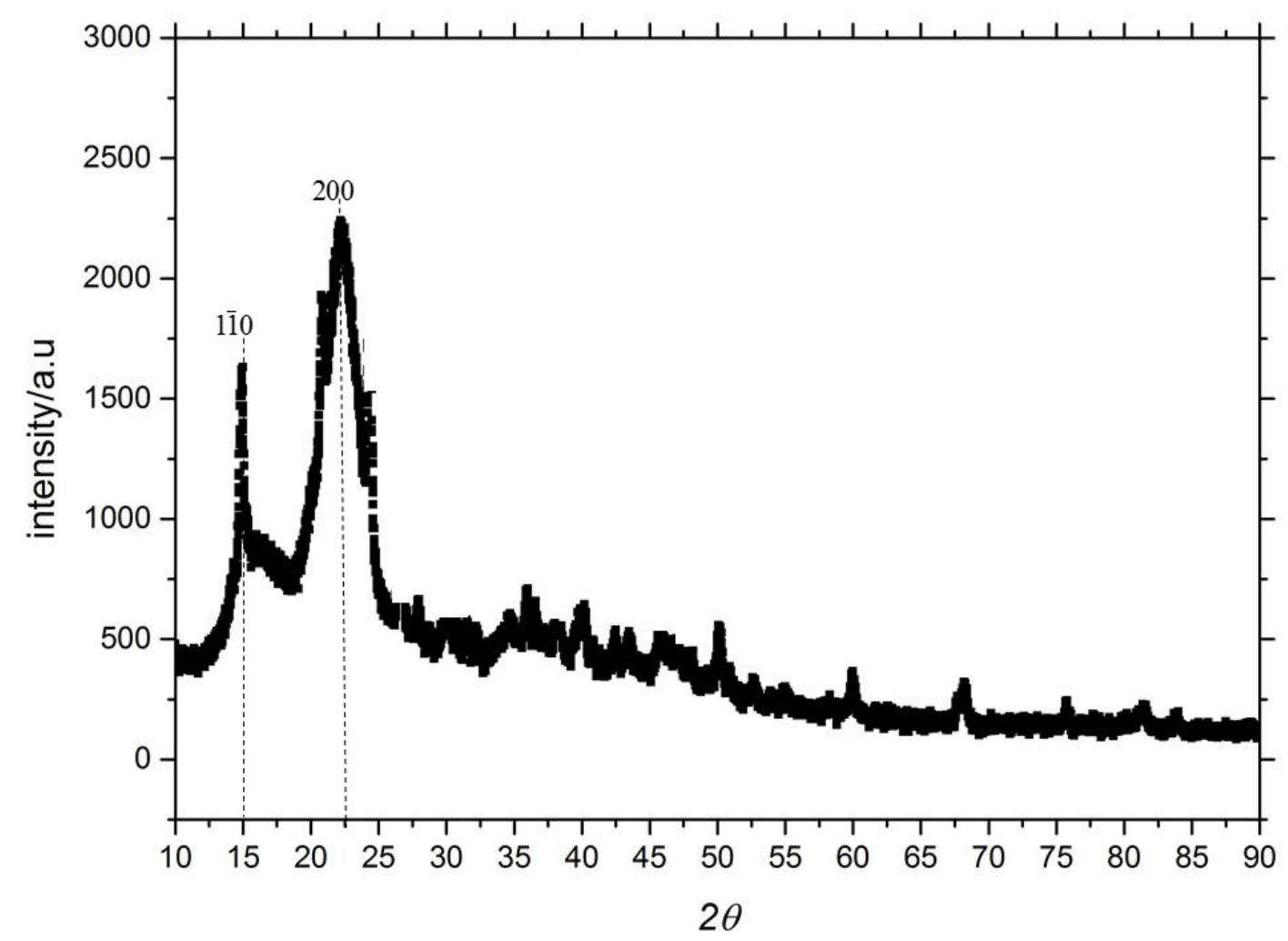

Fig. 3. XRD spectrum of the BNP sheets showing crystalline region at $2 \theta=15$ and amorphous/crystalline regions at $2 \theta=22.5$.

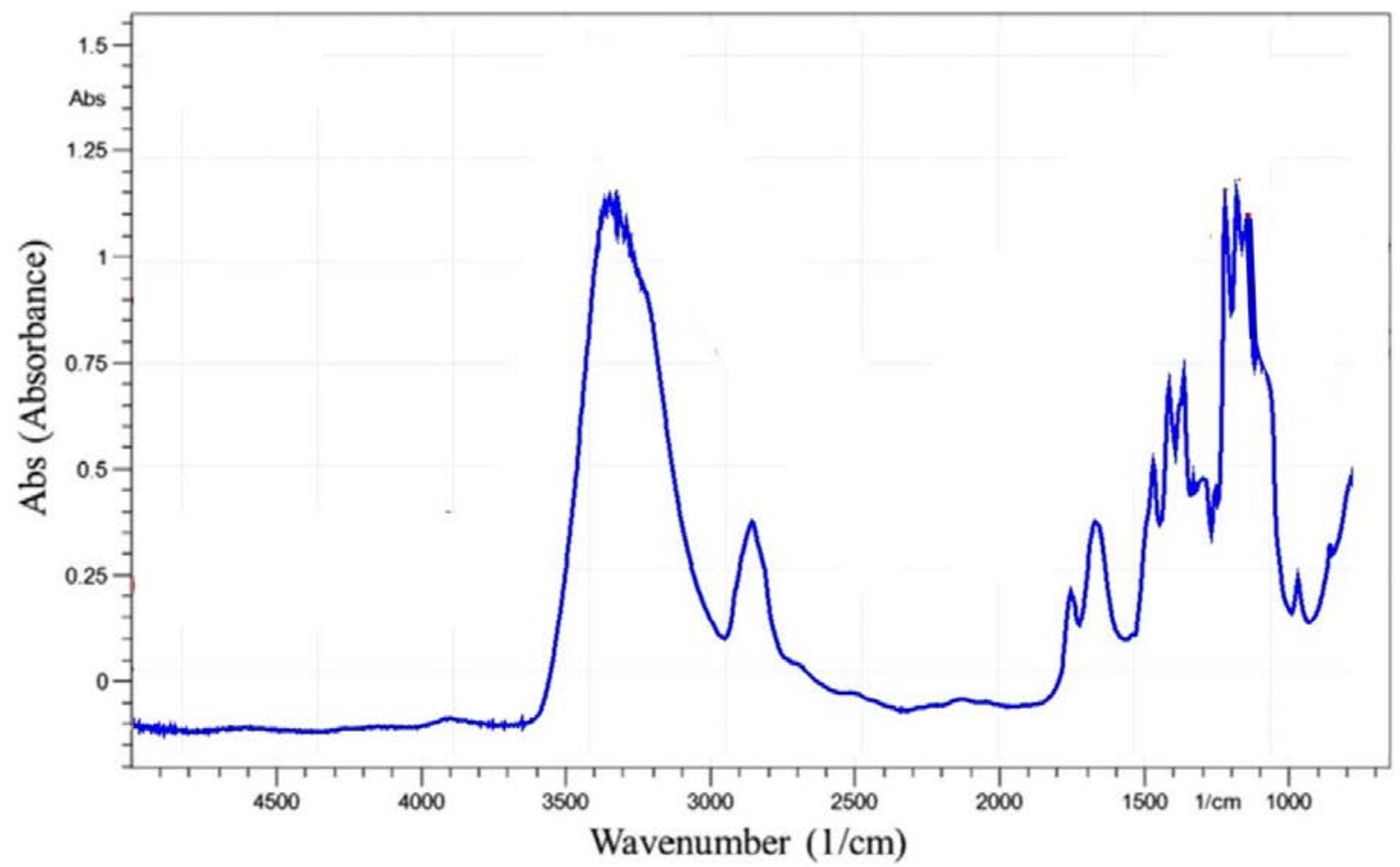

Fig. 4. Diffuse FTIR spectra of the BNP sheets used to determine the their functional groups 


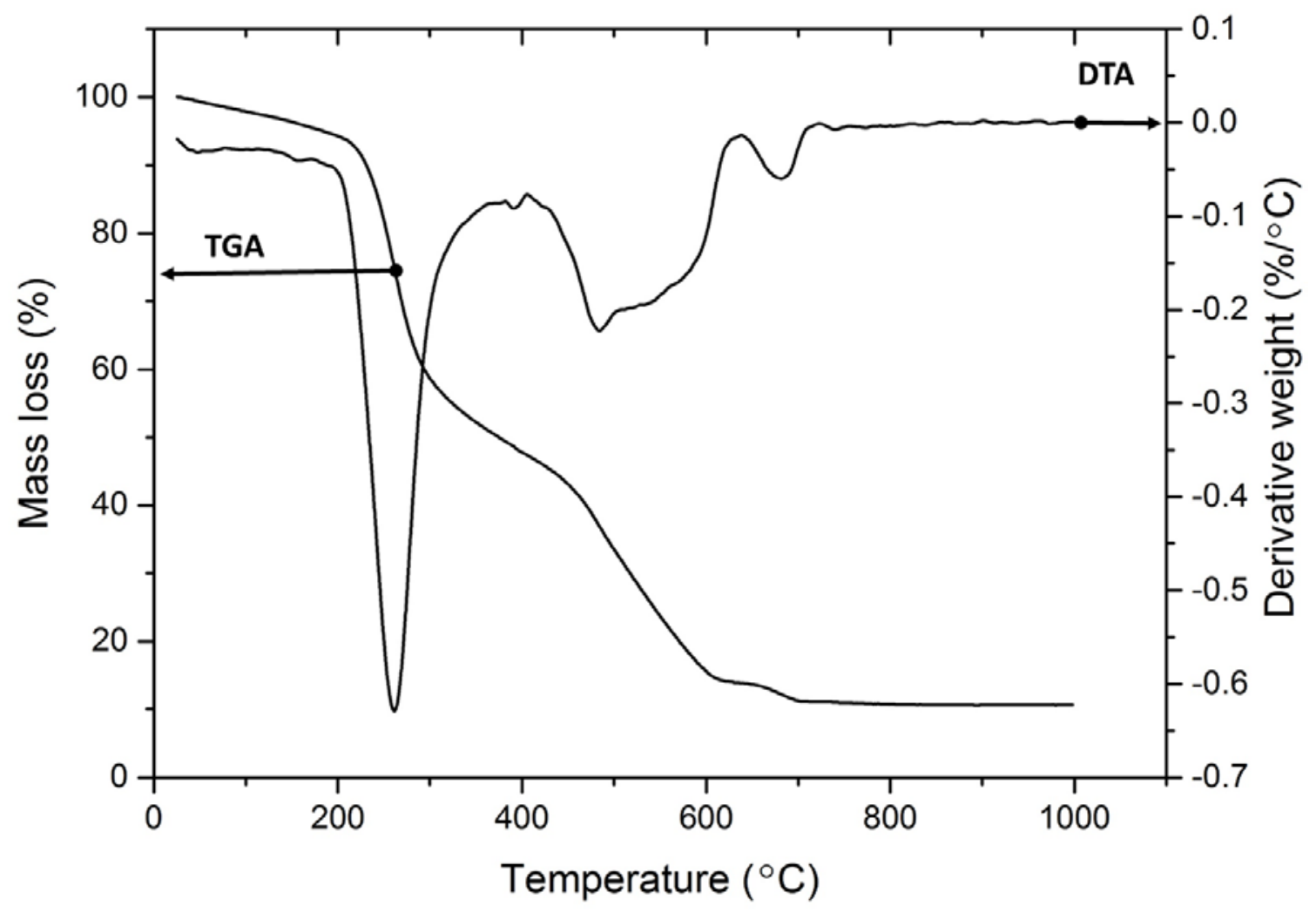

Fig. 5: Thermal stability of the BNP sheets showing TGA and DTA curves

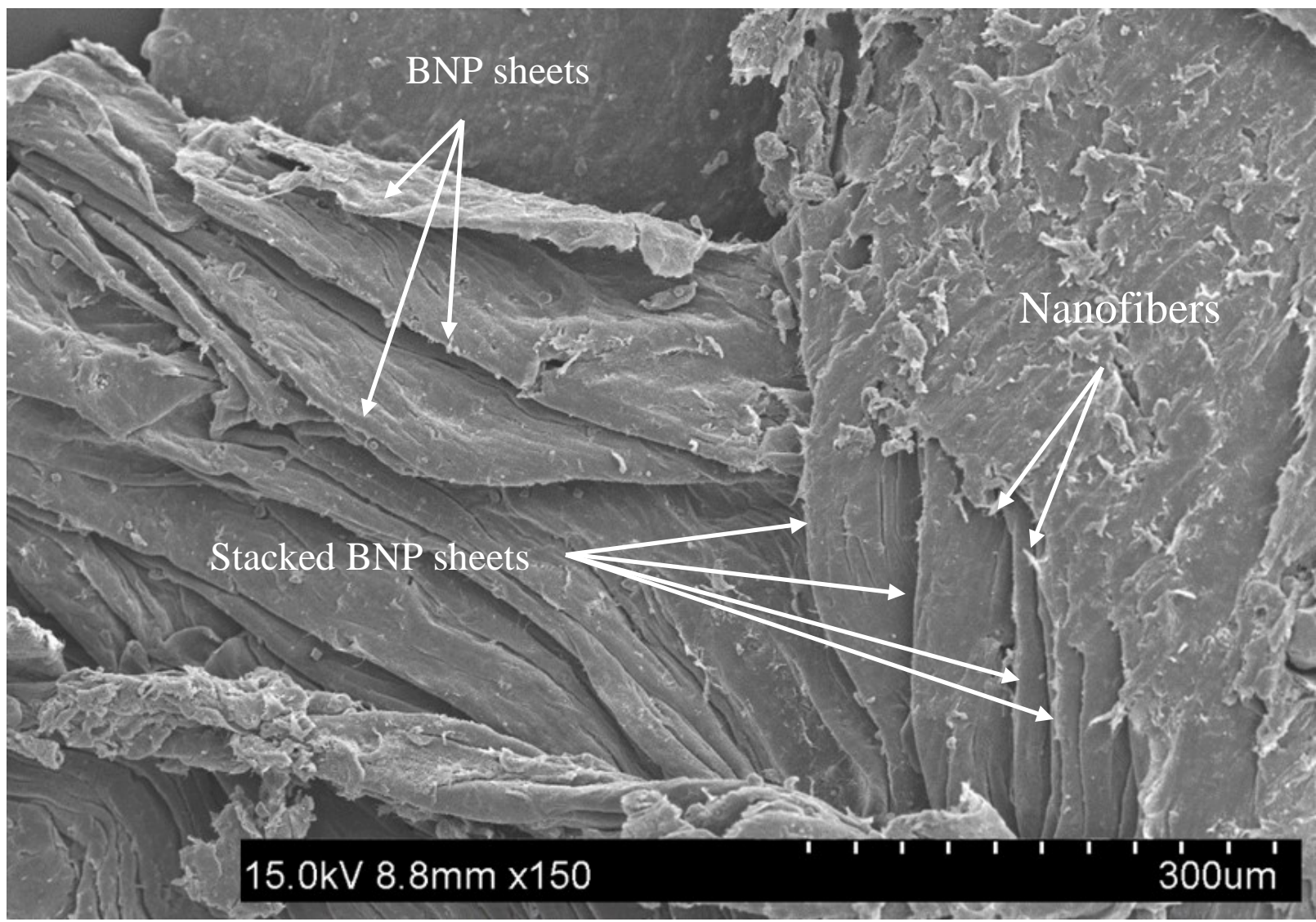

Fig. 6. SEM image showing the surface morphology of the BNP sheets. 
a)

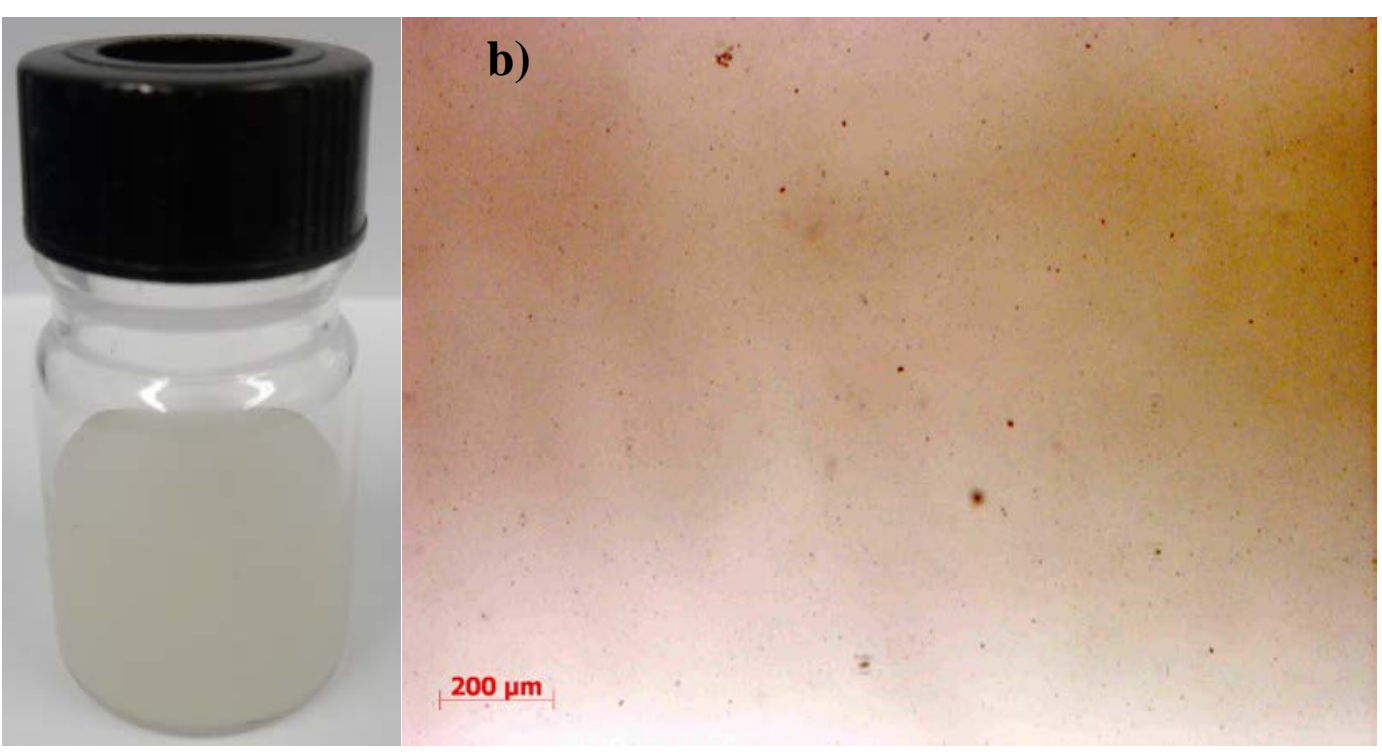

Fig. 7: a) BNP aqueous solution (2g/L) after 30 min of sonication, b) optical image of the BNP aqueous solution.

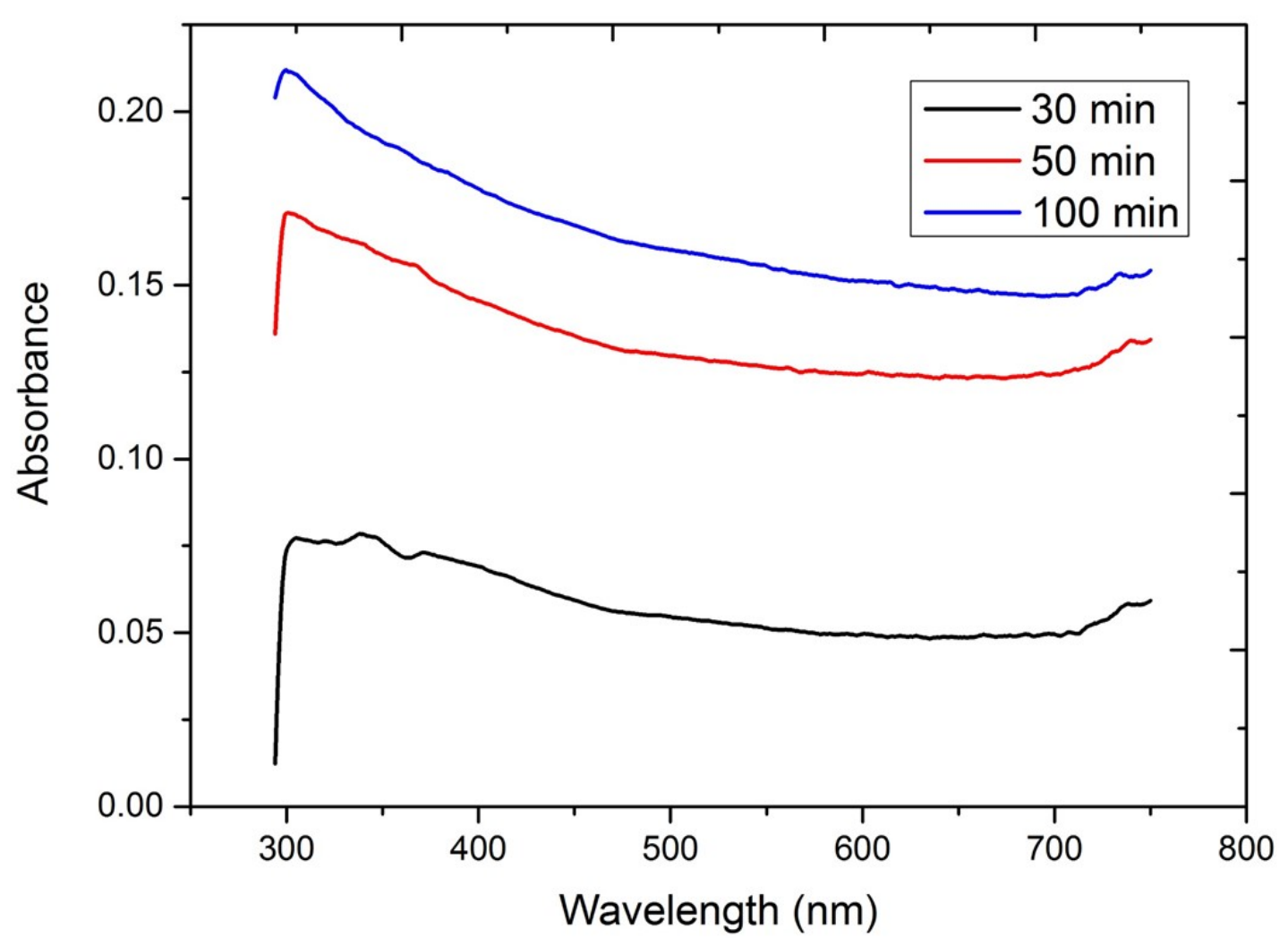

Fig.8. UV-vis spectroscopy results of BNP aqueous solutions at different sonication times 


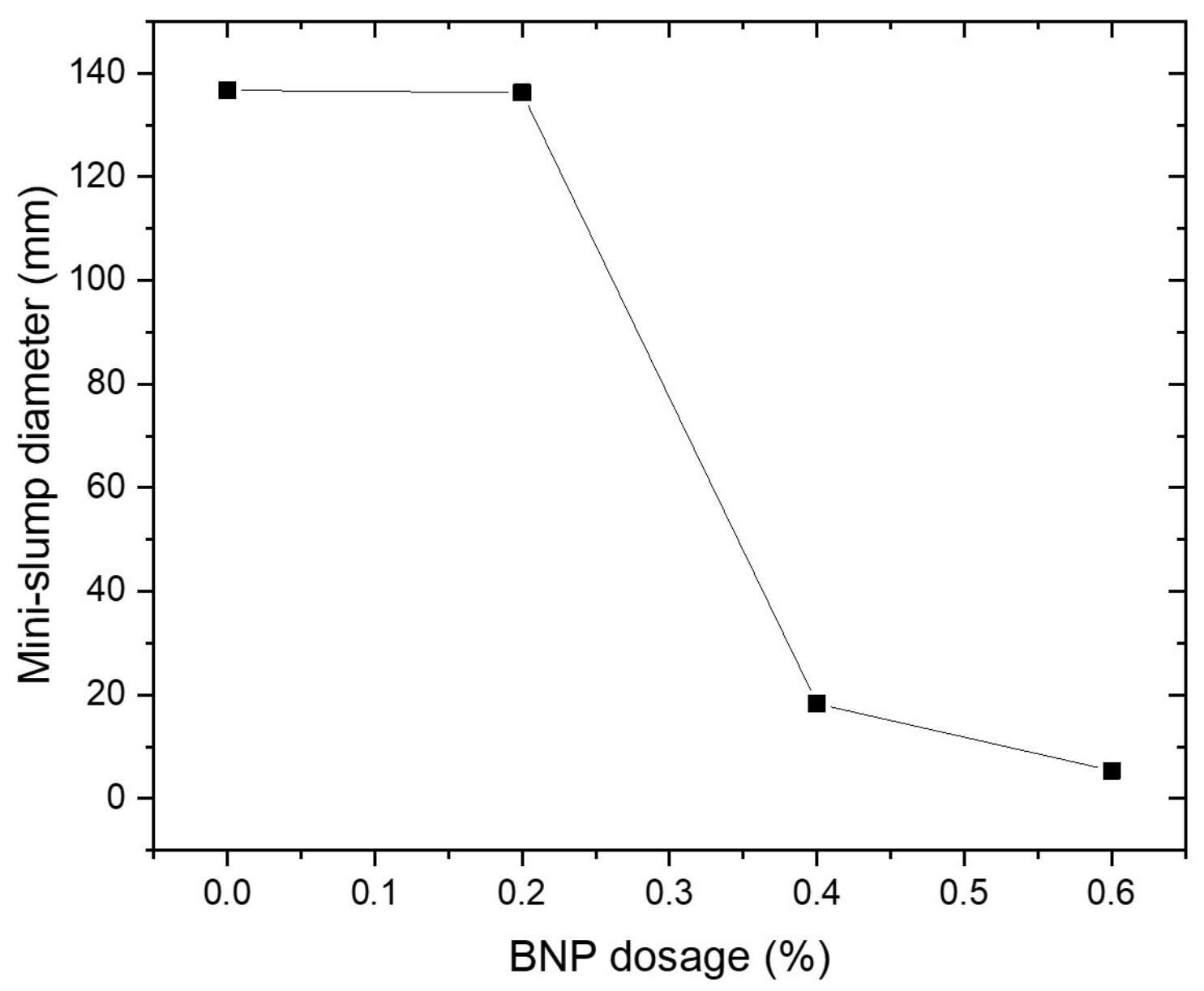

Fig. 9. Effect of the BNP sheets on the workability of the cement paste 

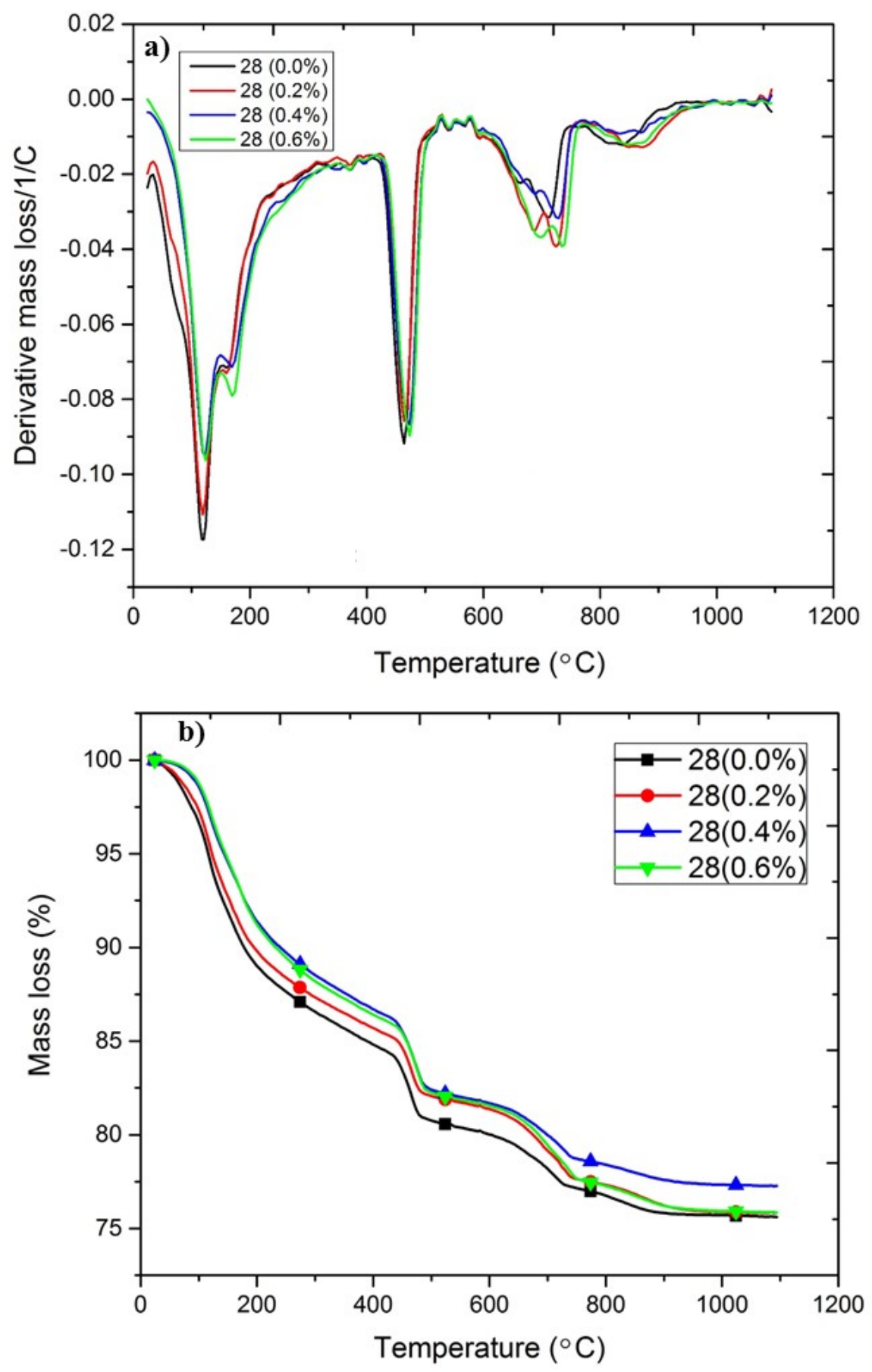

Fig. 10: TGA curves (a) and DTA curves (b) for the cementitious composites at BNP concentrations of 0 (control), 0.2, 0.4 and $0.6-\mathrm{wt} \%$ at 28 days. 


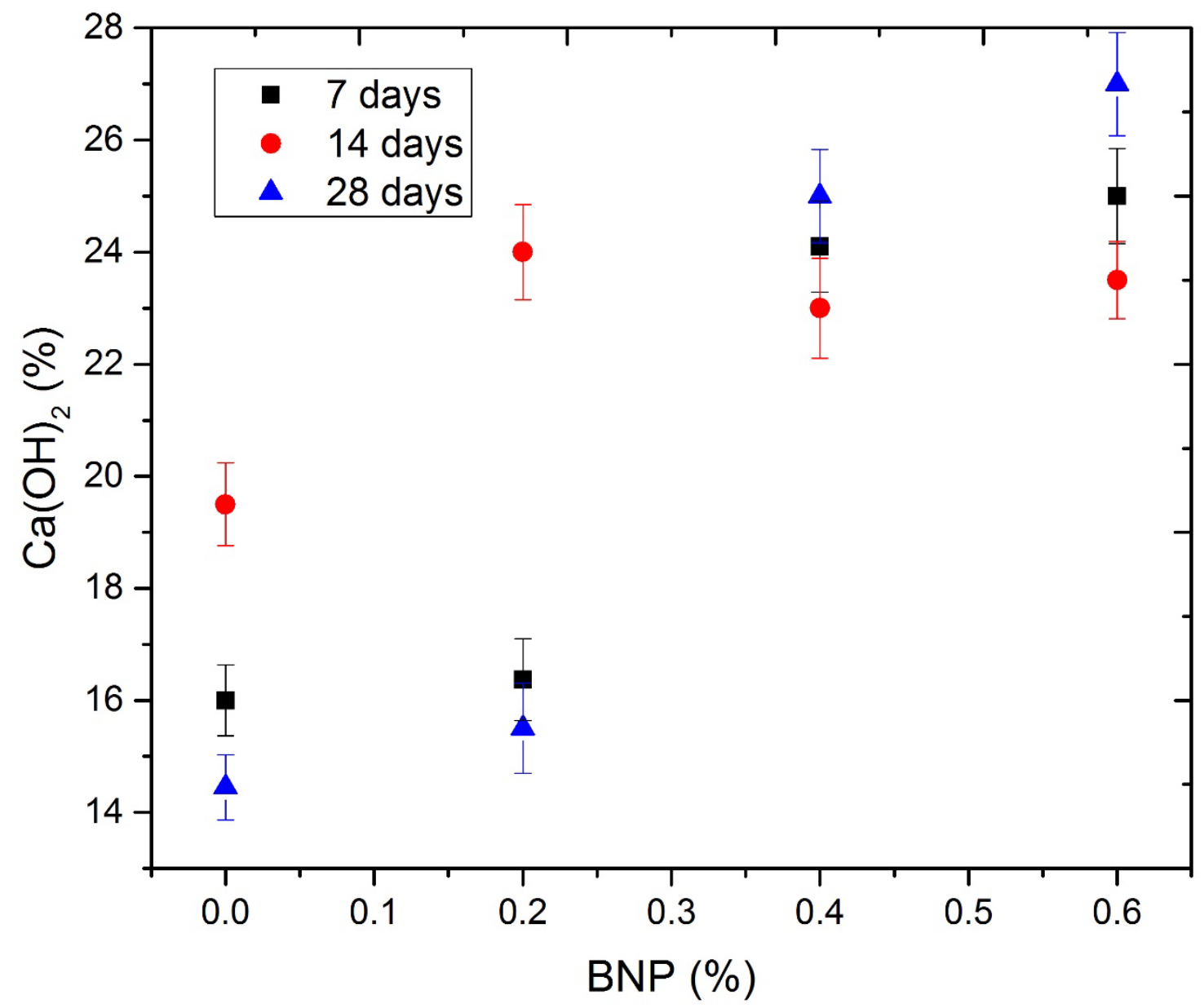

Fig. 11: Content of calcium hydroxide obtained from TGA as a function of BNP concentration at 7, 14 and 28 days

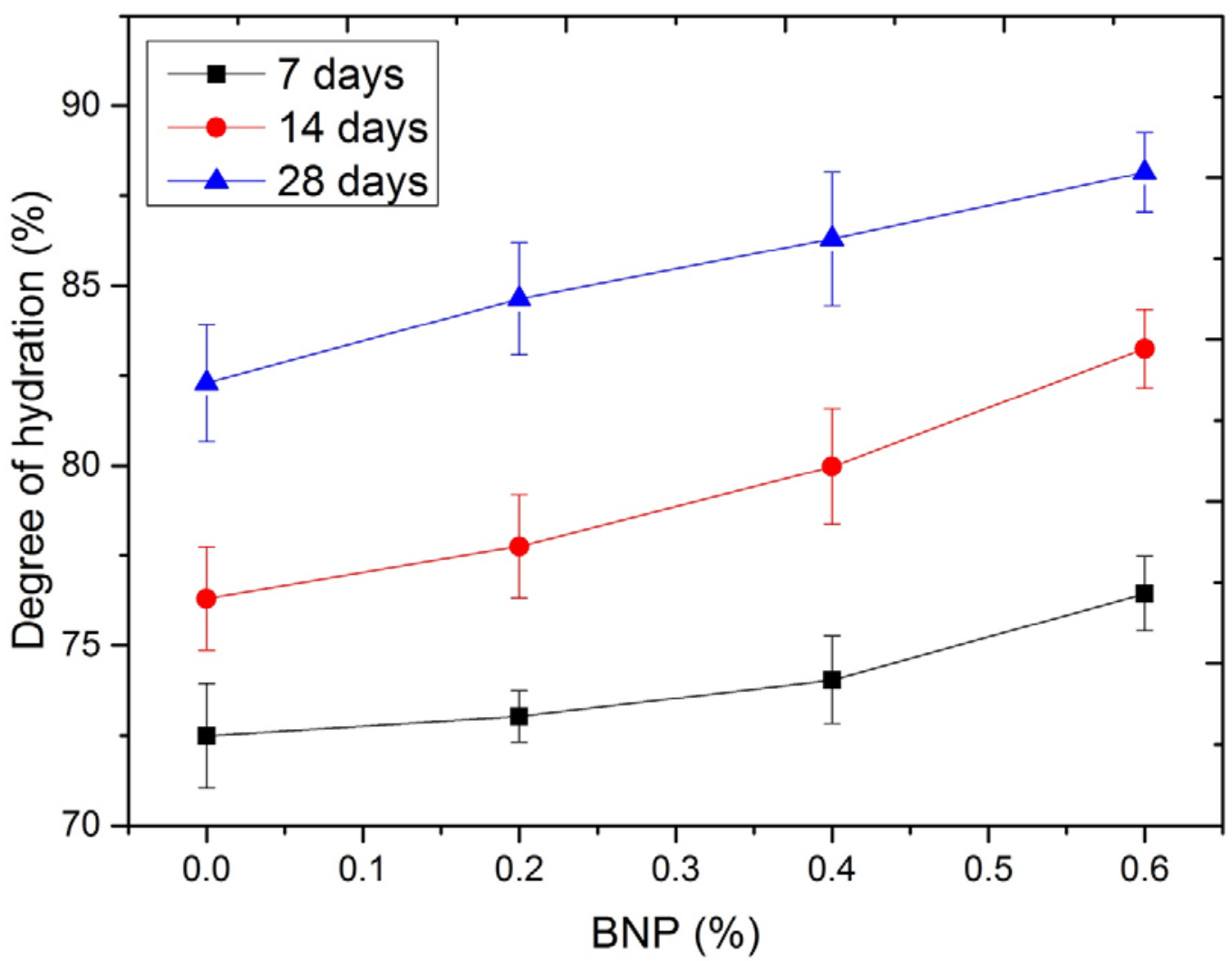

Fig. 12. Degree of hydration (DOH) of the cementitious composites obtained from TGA as a function of BNP concentration at 7, 14 and 28 days. 

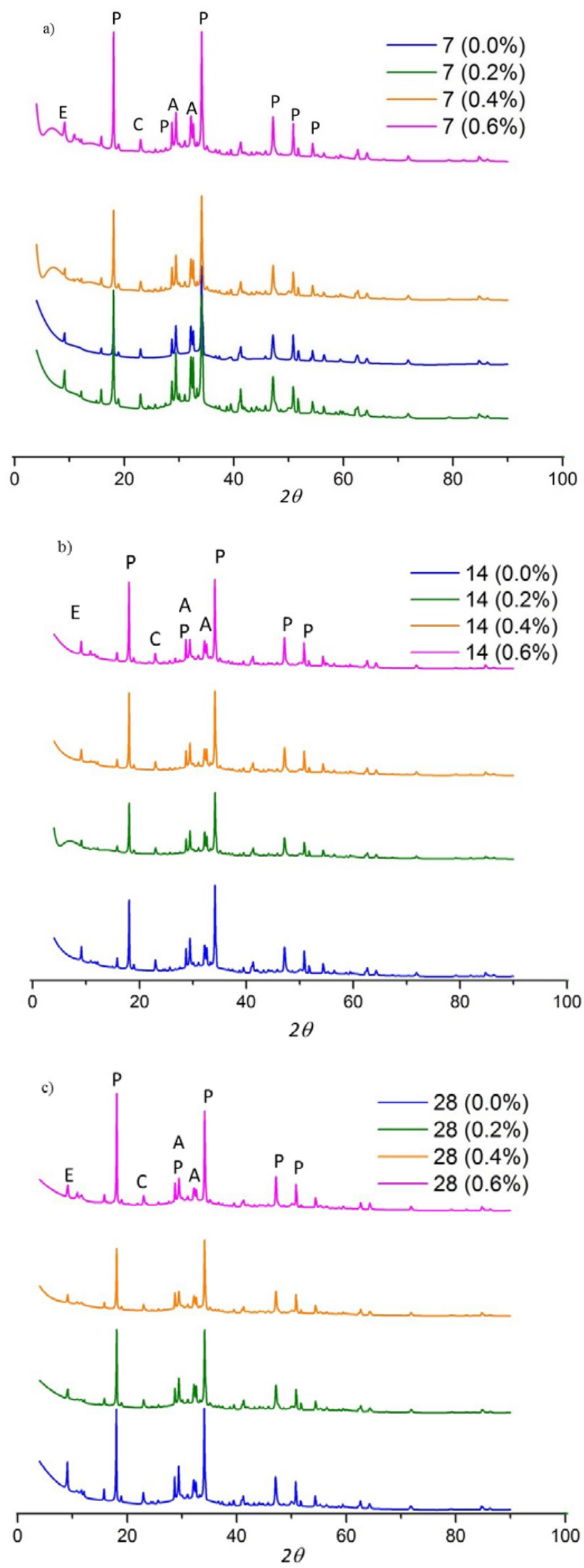

Fig. 13. XRD spectrum of the cementitious composites at different BNP concentrations, a) 7 days, b) 14 days, c) at 28 days. C-S-H: Calcium Silicate hydrate. E: Ettringite, P: Portlandite $\left(\mathrm{Ca}(\mathrm{OH})_{2}\right)$, A: Alite $\left(\mathrm{C}_{3} \mathrm{~S}\right)$, C: Calcite $\left(\mathrm{CaCO}_{3}\right)$. 


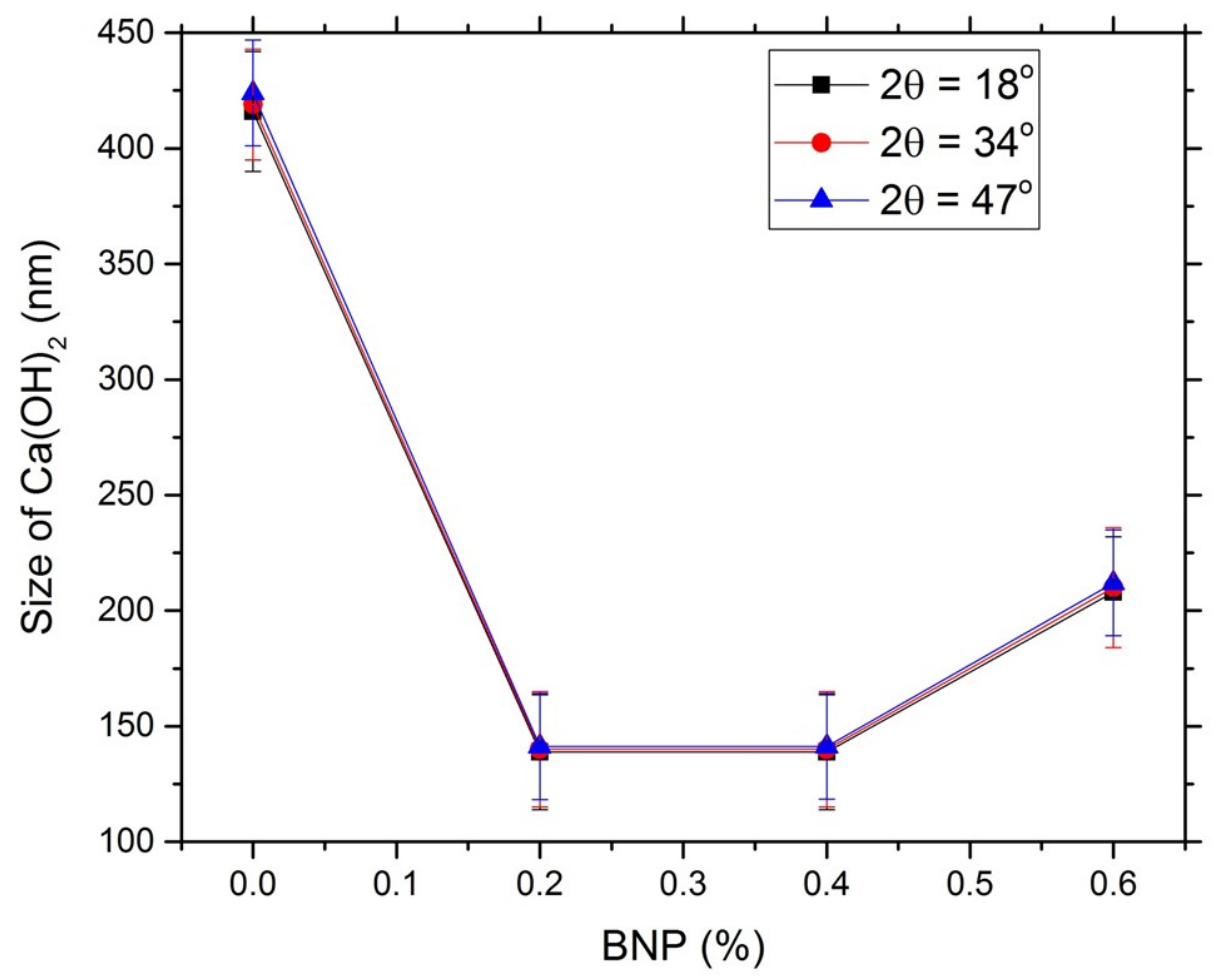

Fig. 14. Size of $\mathrm{Ca}(\mathrm{OH})_{2}$ obtained from XRD as a function of BNP concentration
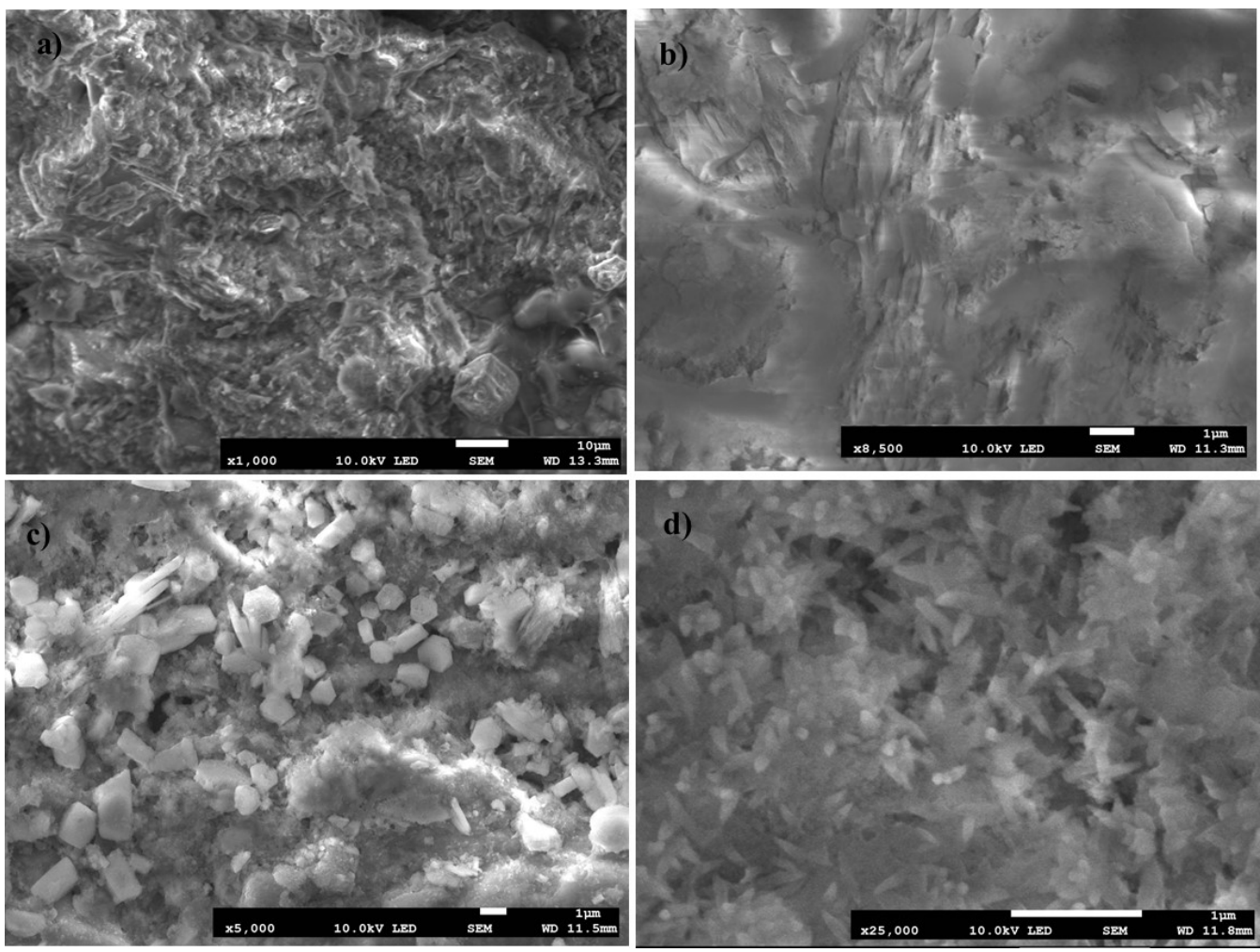

Fig.15. SEM micro images of the cementitious composites at 7 days. a) plain cementitious composite, b) with 0.20-wt\% BNPs, c) with 0.40-wt\% BNPs, d) with 0.60-wt\% BNPs 

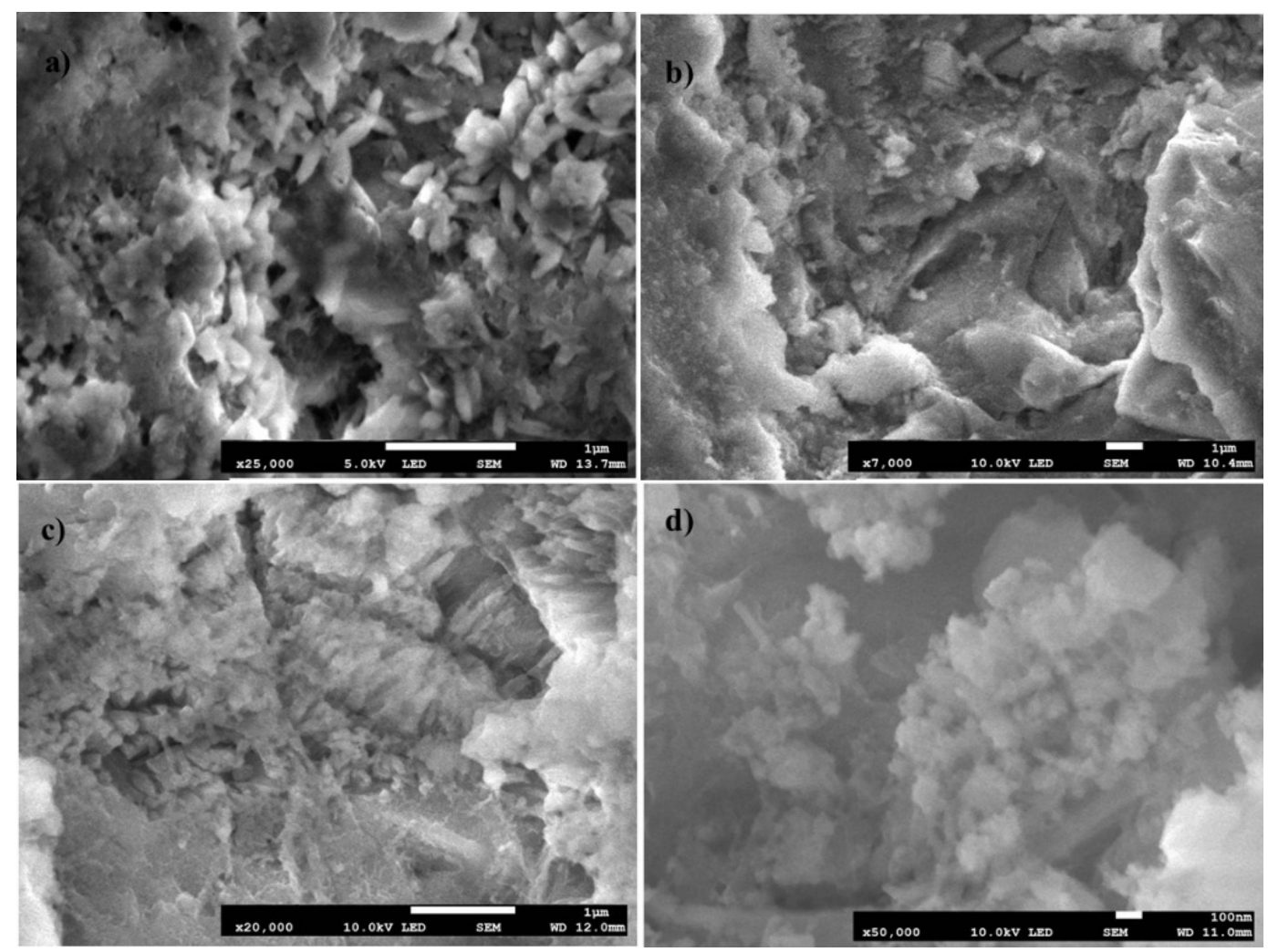

Fig.16. SEM micro images of the cementitious composites at 14 days. a) plain cementitious composite, b) with 0.20-wt\% BNPs, c) with 0.40-wt\% BNPs, d) with 0.60-wt\% BNPs 

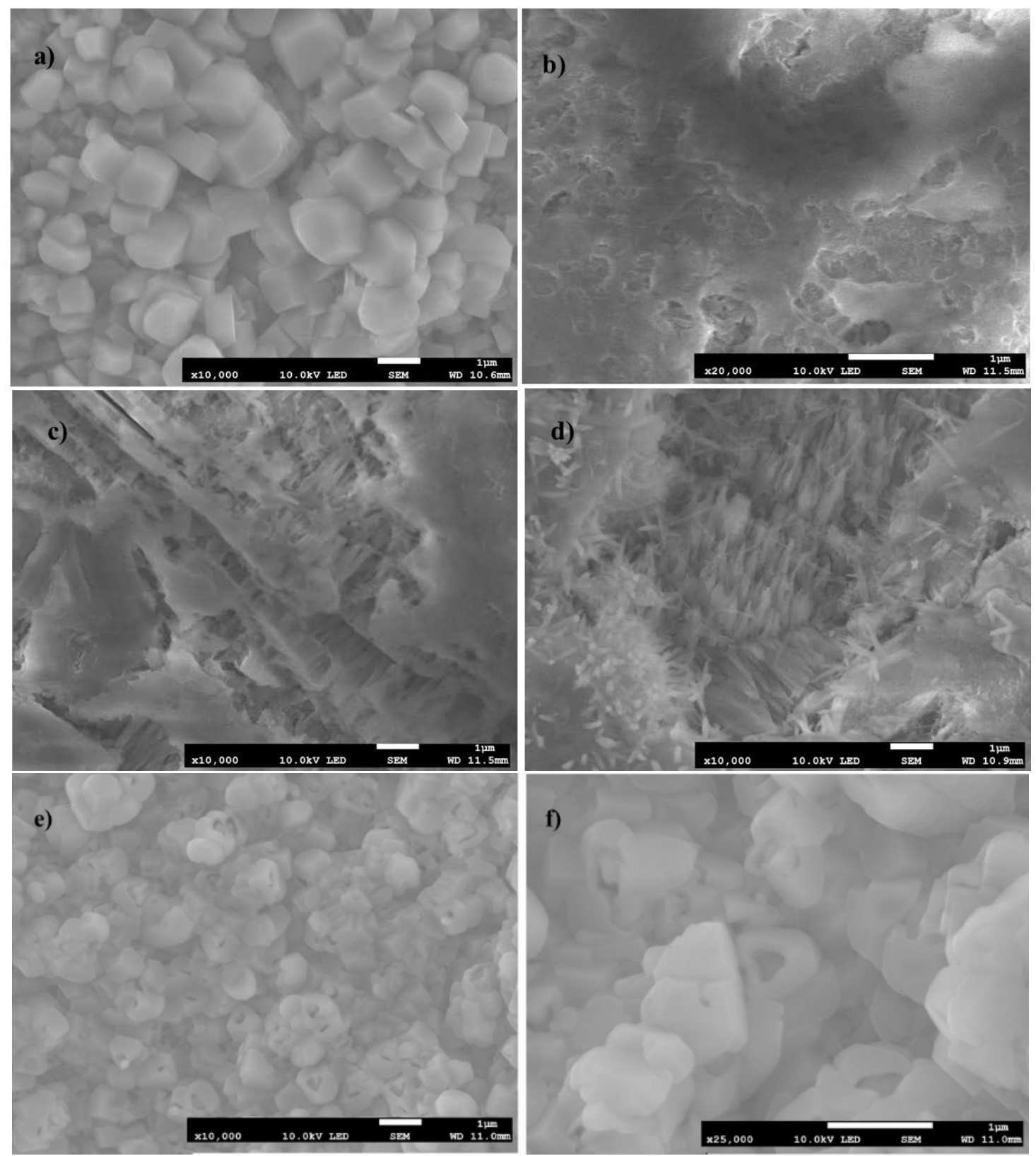

Fig.17. SEM micro images of the cementitious composites at 28 days. a) plain cementitious composite, b,c) with 0.20 -wt\% BNPs, d) with 0.40-wt\% of BNPs, e,f) with 0.60 -wt\% of BNPs 

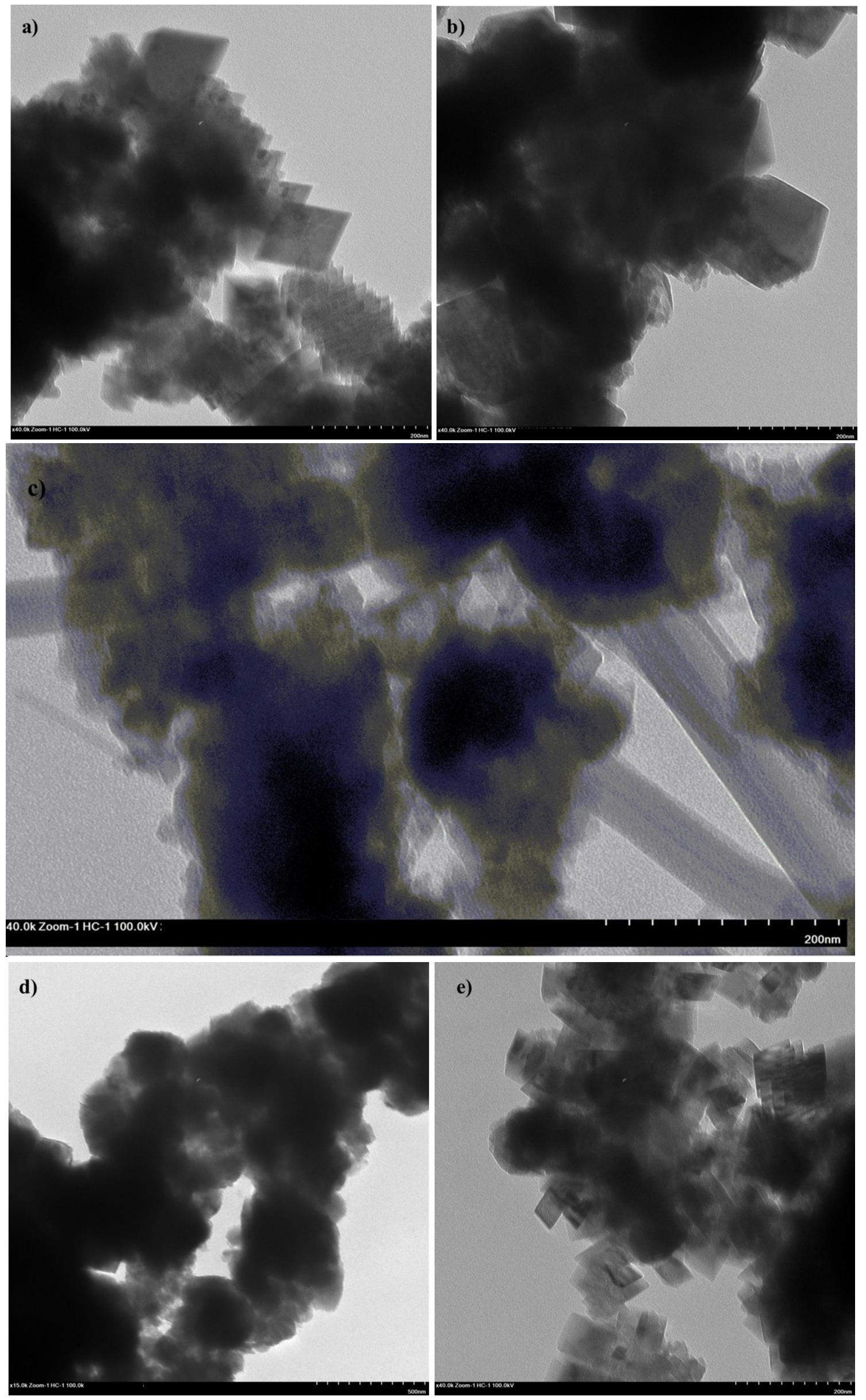

Fig. 18. TEM micro images at 28 days of curing a) plain cementitious composite, b,c) with 0.20 -wt\% BNPs, c) with 0. 20-wt\% BNPs showing fabrics-like crystals, d) with 0.40-wt\% BNPs, e) with 0.60-wt\% BNPs 


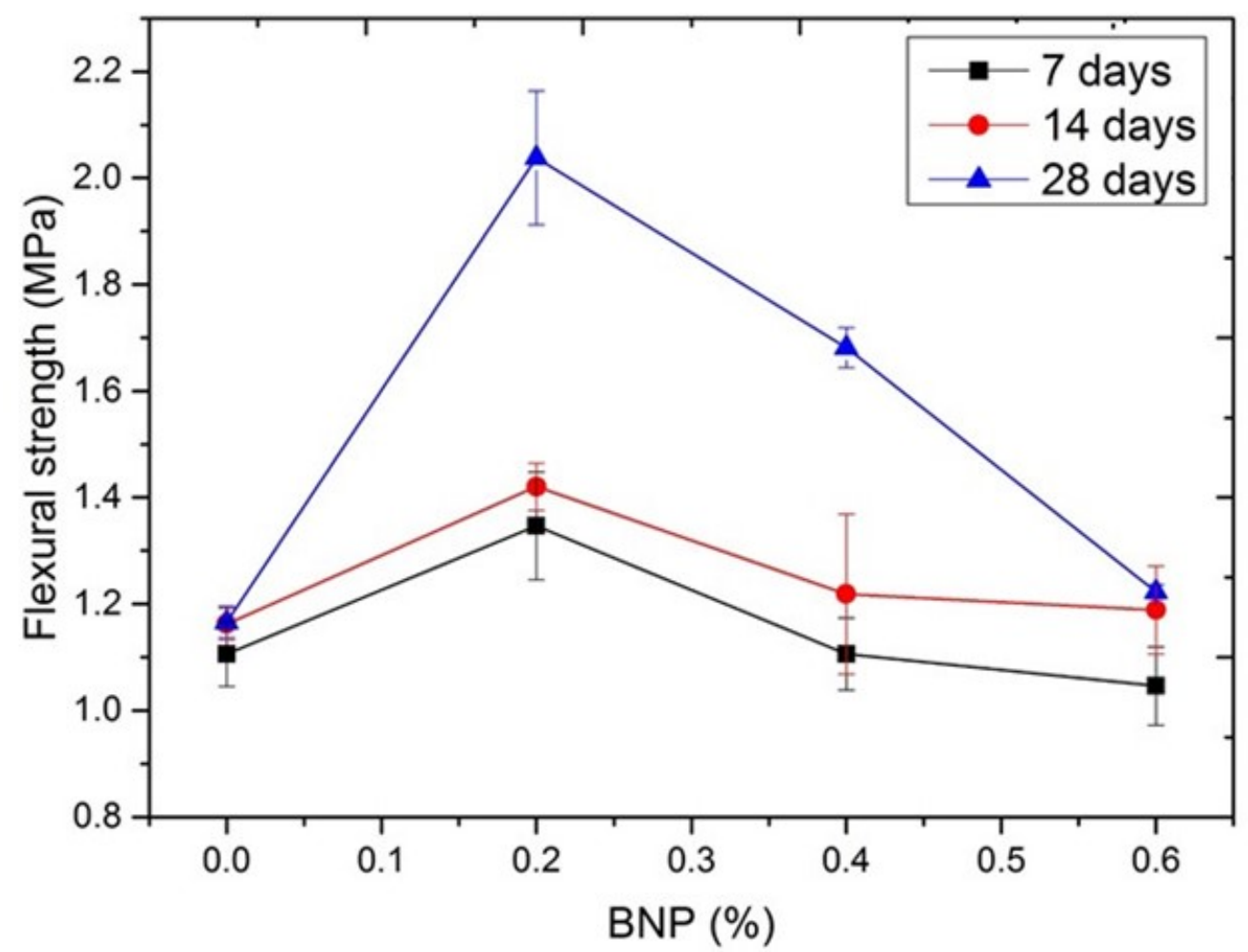

Fig. 19. Variation of the flexural strength as a function of BNP concentration at different curing ages

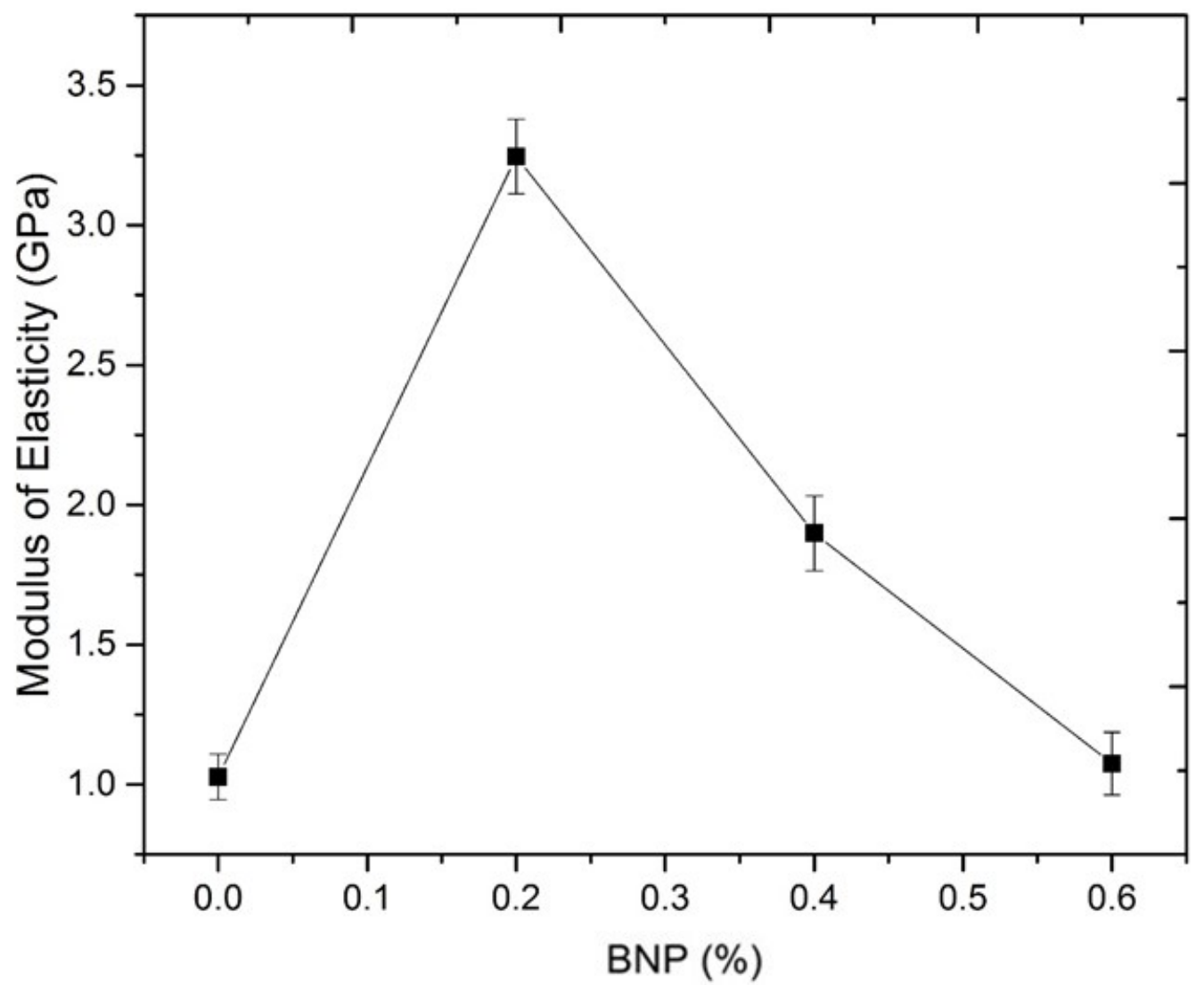

Fig. 20. Variation of the modulus of elasticity as a function of BNP concentration at 28 days 


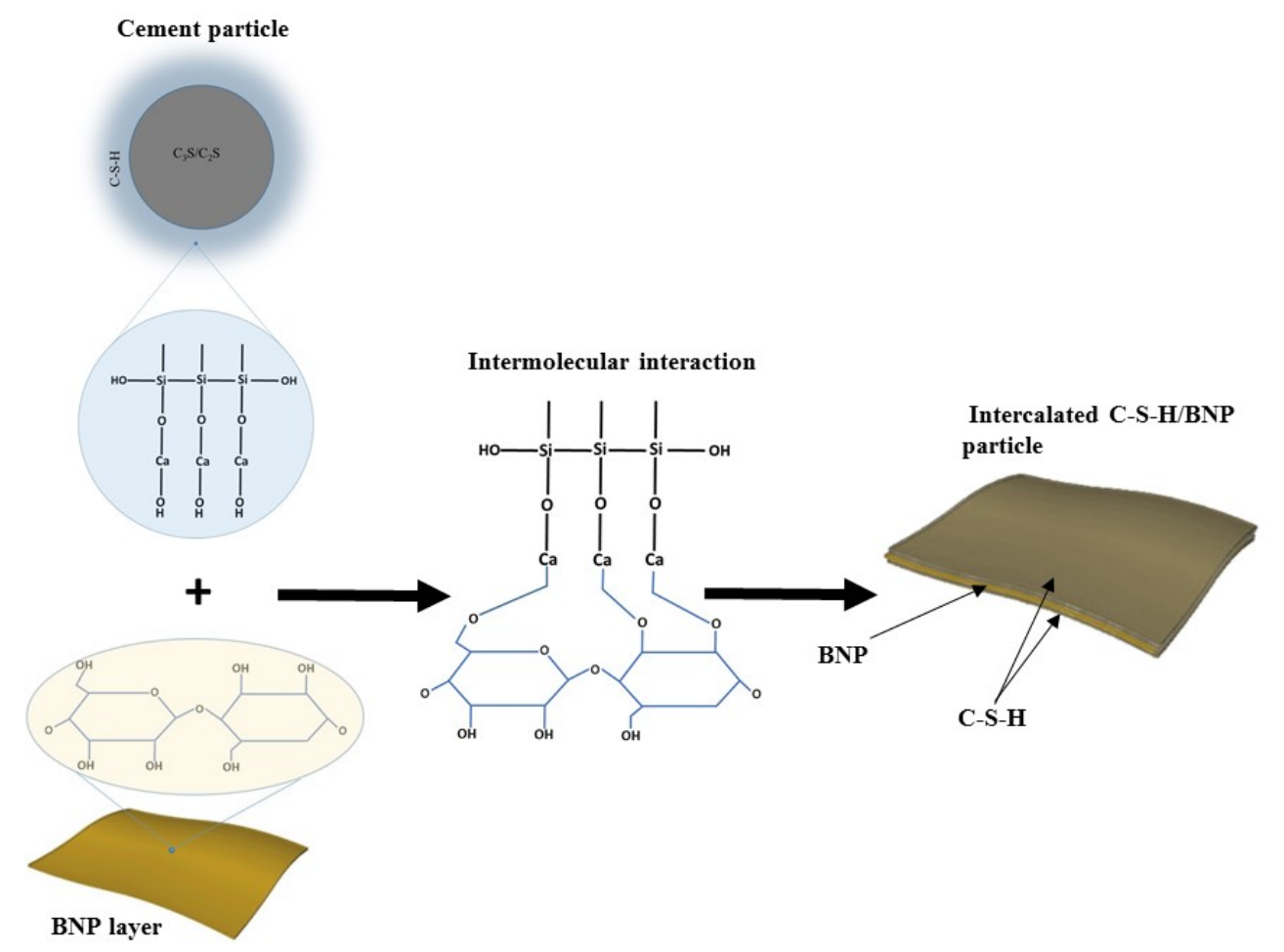

Fig. 21. Schematic illustration of intermolecular interaction of BPN with C-S-H phase at 28 days.

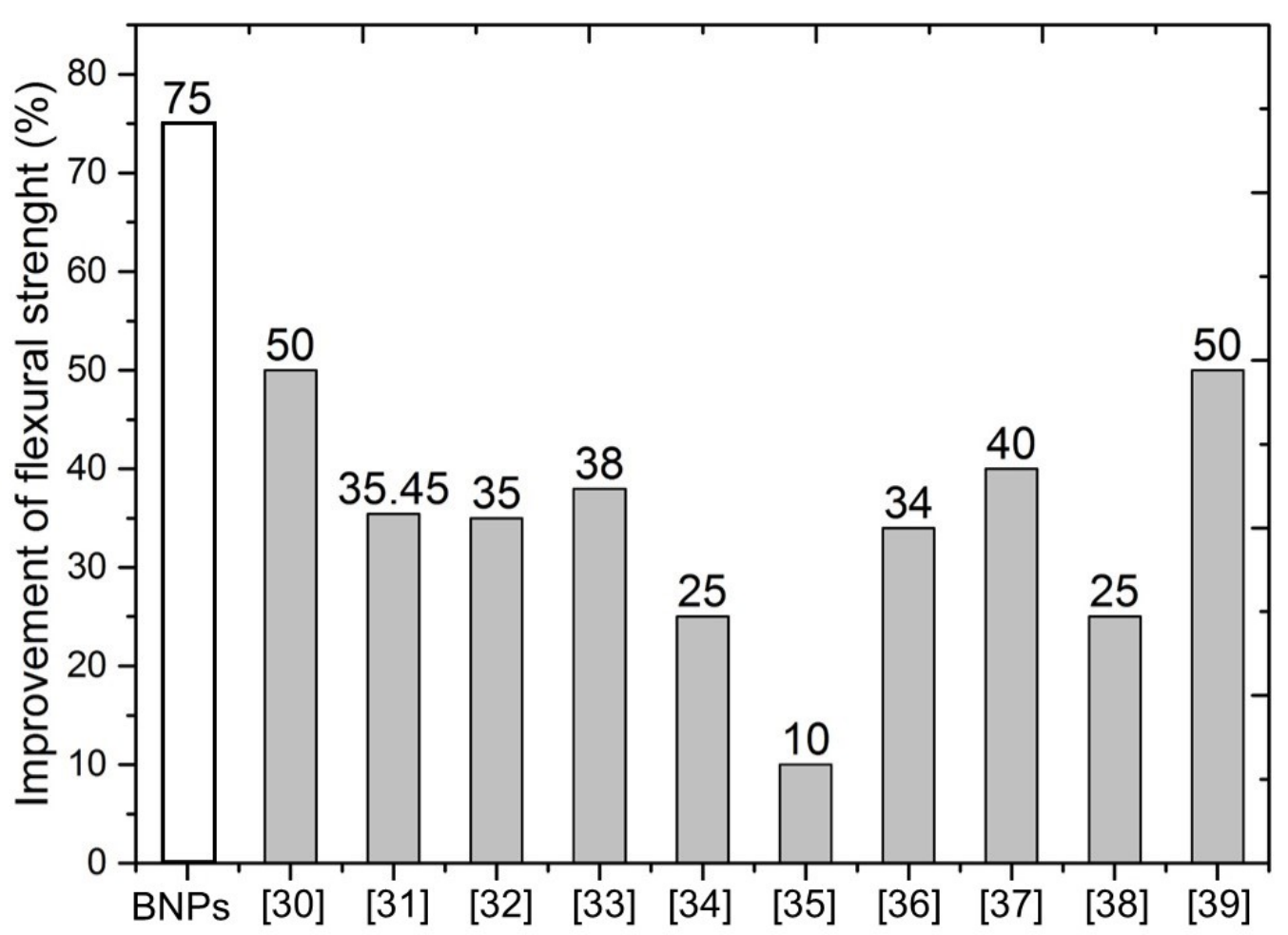

Fig. 22. Comparison of maximum flexural strength of BNP-cementitious composites and MWCNTcementitious composites from References [30-39] in terms of percentage increase. 


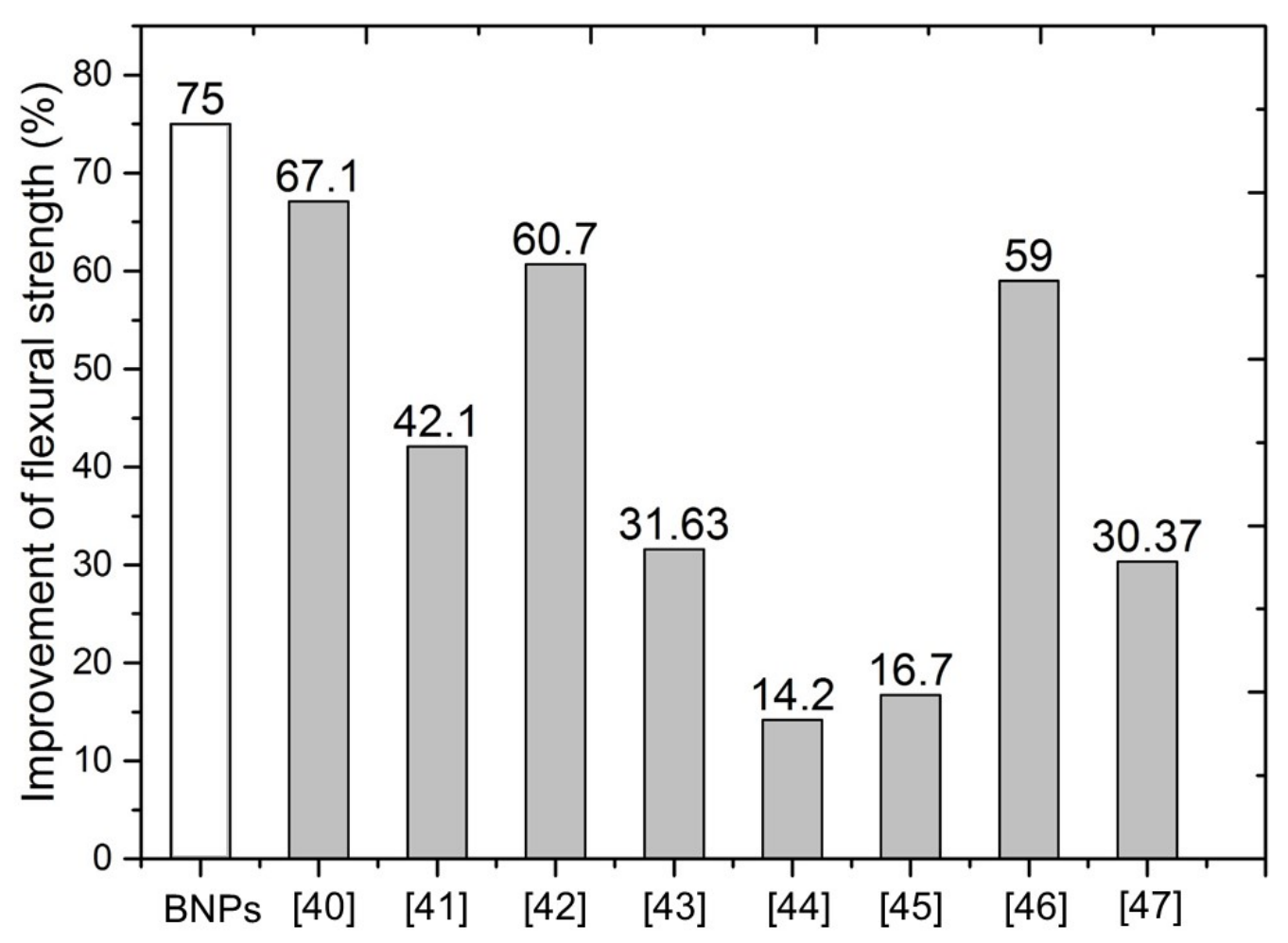

Fig. 23. Comparison of maximum flexural strength of BNP-cementitious composites and GO-cementitious composites from References [41-48] in terms of percentage increase.

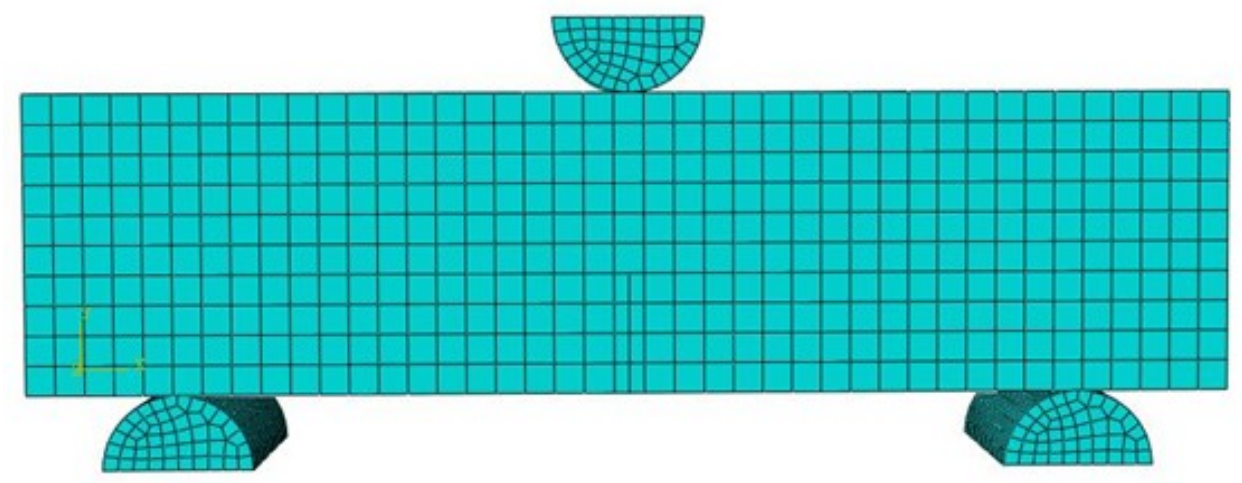

Fig. 24. X-FEM model of the notched cementitious composite prism for fracture modelling and analysis

Table 2.Average material properties of the cementitious composites

\begin{tabular}{lccc}
\hline Material & $\begin{array}{c}\text { Flexural strength } \\
(\mathrm{MPa})\end{array}$ & $\begin{array}{c}\text { Modulus of Elasticity } \\
(\mathrm{GPa})\end{array}$ & $\begin{array}{c}\text { Fracture Energy } \\
(\mathrm{N} / \mathrm{M})\end{array}$ \\
\hline Plain cement & 1.2 & 1.12 & 12.55 \\
\hline With 0.20-wt\% BNPs & 2.1 & 3.25 & 23.60 \\
\hline
\end{tabular}




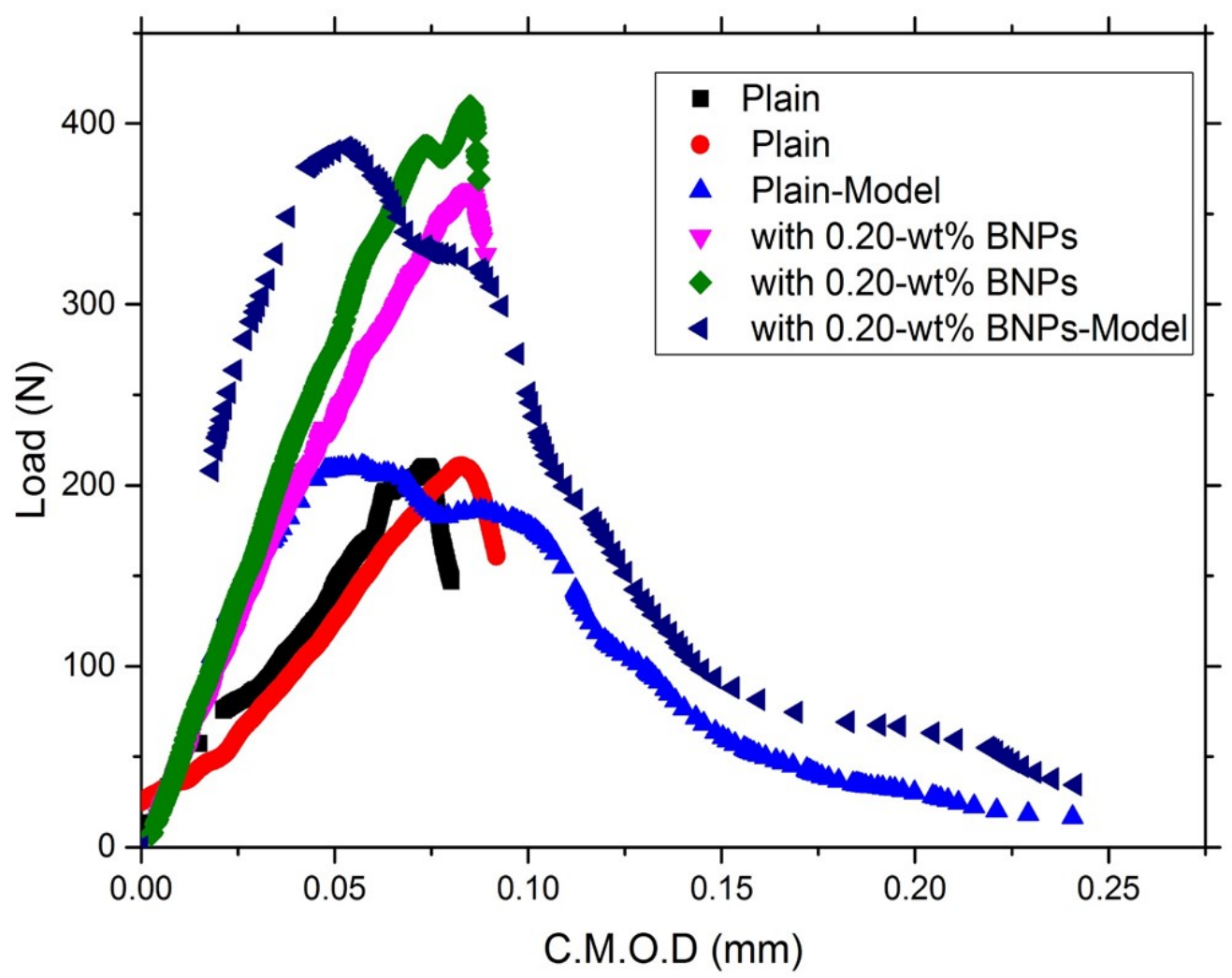

Fig. 25. Load versus crack mouth opening displacement for cementitious composites with and without 0.20wt\% BNPs. experimental versus modelling.
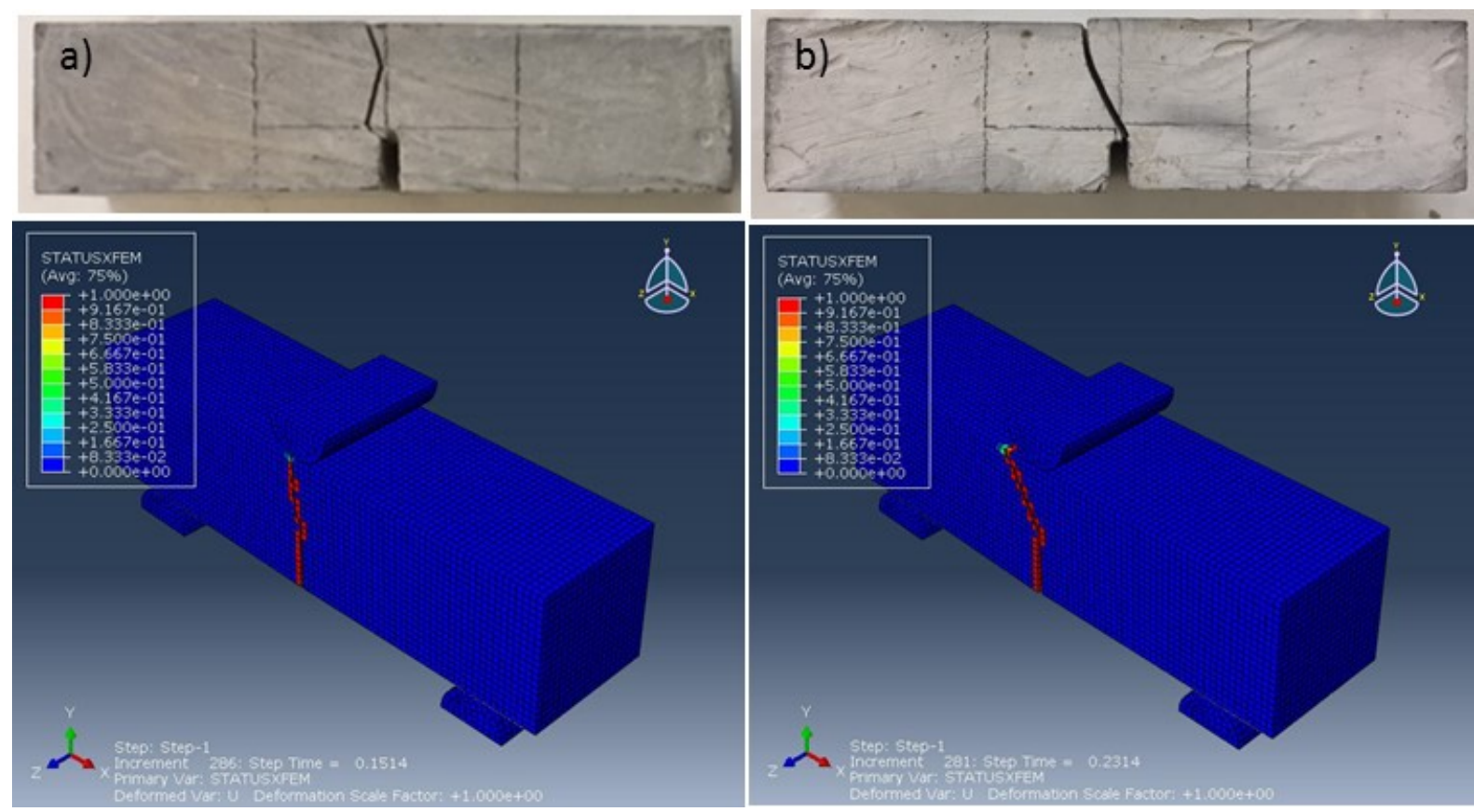

Figure 26. Experimental and simulated fracture behavior of the cementitious composite beams. a) plain cement prism, b) with 0.20-wt\% BNPs. 

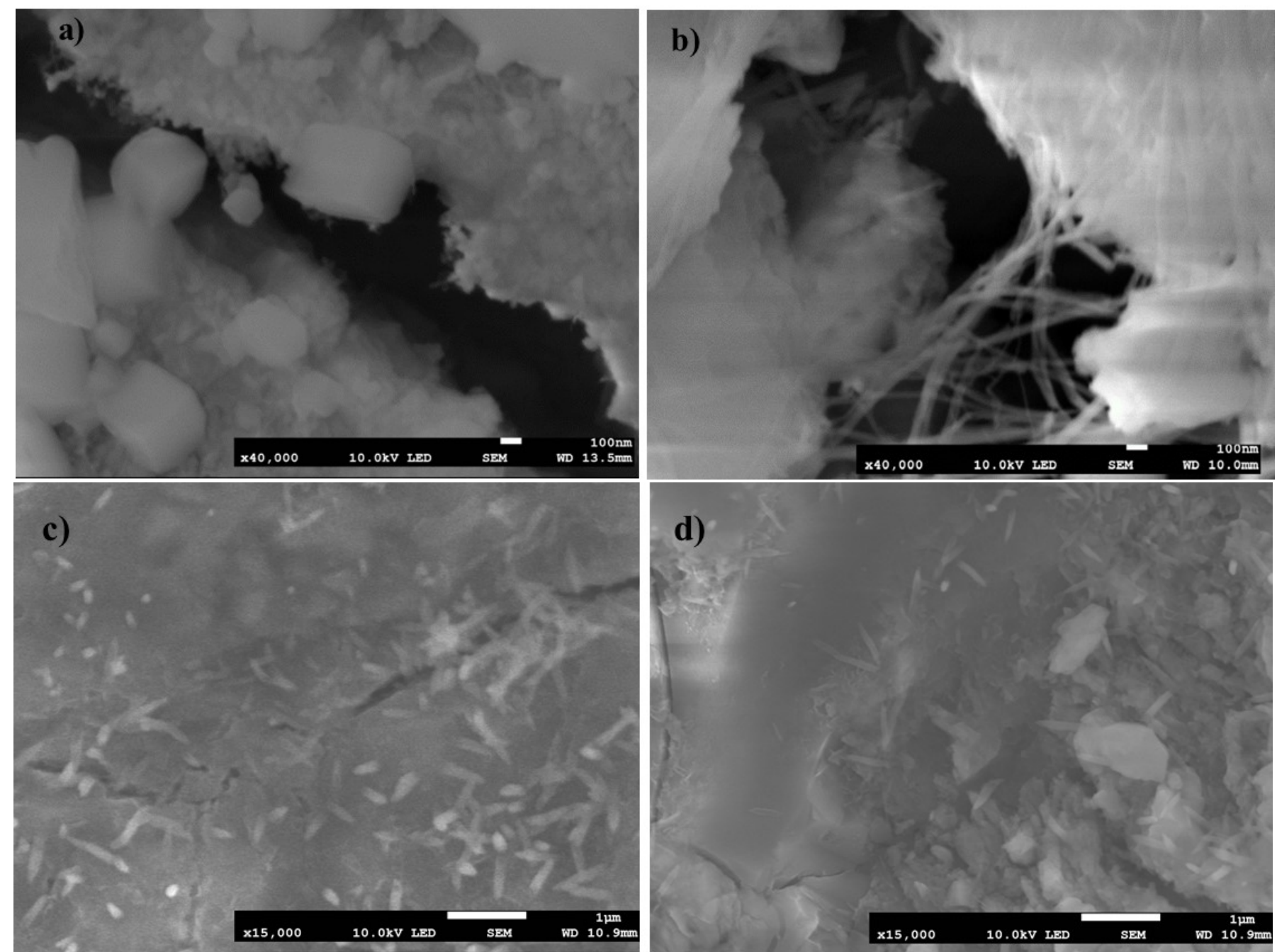

Fig. 27. SEM micro image showing crack propagation in a) plain cementitious composite, b) with BNPs showing BNP crack bridging, $c$ and d) with BNP showing crack deflection 